FACTA UNIVERSITATIS (NIŠ)

Ser. Math. Inform. Vol. 35, No 1 (2020), 217-242

https://doi.org/10.22190/FUMI2001217Z

\title{
GENERALIZED BESSEL AND FRAME MEASURES
}

\author{
Fariba Zeinal Zadeh Farhadi, Mohammad Sadegh Asgari, \\ Mohammad Reza Mardanbeigi and Mahdi Azhini
}

(c) 2020 by University of Niš, Serbia | Creative Commons License: CC BY-NC-ND

\begin{abstract}
Considering a finite Borel measure $\mu$ on $\mathbb{R}^{d}$, a pair of conjugate exponents $p, q$, and a compatible semi-inner product on $L^{p}(\mu)$, we have introduced $(p, q)$-Bessel and $(p, q)$-frame measures as a generalization of the concepts of Bessel and frame measures. In addition, we have defined the notions of $q$-Bessel sequence and $q$-frame in the semi-inner product space $L^{p}(\mu)$. Every finite Borel measure $\nu$ is a $(p, q)$-Bessel measure for a finite measure $\mu$. We have constructed a large number of examples of finite measures $\mu$ which admit infinite $(p, q)$-Bessel measures $\nu$. We have showed that if $\nu$ is a $(p, q)$-Bessel/frame measure for $\mu$, then $\nu$ is $\sigma$-finite and it is not unique. In fact, by using the convolutions of probability measures, one can obtain other $(p, q)$-Bessel/frame measures for $\mu$. We have presented a general way of constructing a $(p, q)$-Bessel/frame measure for a given measure.
\end{abstract}

Keywords: Fourier frame, Plancherel theorem, spectral measure, frame measure, Bessel measure, semi-inner product.

\section{Introduction}

According to [5], a Borel measure $\nu$ on $\mathbb{R}^{d}$ is called a dual measure for a given measure $\mu$ on $\mathbb{R}^{d}$ if for every $f \in L^{2}(\mu)$,

$$
\int_{\mathbb{R}^{d}}|\widehat{f d \mu}(t)|^{2} d \nu(t) \simeq \int_{\mathbb{R}^{d}}|f(x)|^{2} d \mu(x),
$$

where for a function $f \in L^{1}(\mu)$ the Fourier transform is given by

$$
\widehat{f d \mu}(t)=\int_{\mathbb{R}^{d}} f(x) e^{-2 \pi i t \cdot x} d \mu(x) \quad\left(t \in \mathbb{R}^{d}\right) .
$$

Precisely, the equivalence in equation (1.1) means that there are positive constants $A$ and $B$ independent of the function $f(x)$ such that

$$
A \int_{\mathbb{R}^{d}}|f(x)|^{2} d \mu(x) \leq \int_{\mathbb{R}^{d}}|\widehat{f d \mu}(t)|^{2} d \nu(t) \leq B \int_{\mathbb{R}^{d}}|f(x)|^{2} d \mu(x) .
$$

Received

2010 Mathematics Subject Classification. Primary 28A99; Secondary 46E30, 42C15 
Therefore, when $A=B=1$, by Plancherel's theorem for Lebesgue measure $\lambda$ on $\mathbb{R}^{d}, \lambda$ is a dual measure to itself. Dual measures are in fact a generalization of the concept of Fourier frames and they are also called frame measures. According to [5], if $\mu$ is not an $F$-spectral measure (see Definition 2.3), then there cannot be any general statement about the existence of frame measures $\nu$. Nevertheless, the authors showed that if one frame measure exists, then by using convolutions of measures, many frame measures can be obtained, especially a frame measure which is absolutely continuous with respect to Lebesgue measure. Moreover, they presented a general way of constructing Bessel/frame measures for a given measure.

In this paper, we generalize the notion of Bessel/frame measure from Hilbert spaces $L^{2}(\mu), L^{2}(\nu)$ to Banach spaces $L^{p}(\mu), L^{q}(\nu)$ ( $p, q$ are conjugate exponents) via a compatible semi-inner product defined on $L^{p}(\mu)$. Compatible semi-inner products are natural substitutes for inner products on Hilbert spaces. We introduce $(p, q)$-Bessel and $(p, q)$-frame measures, and we define notions of $q$-Bessel sequence and $q$-frame in the semi-inner product space $L^{p}(\mu)$. Then we investigate the existence and some general properties of them.

The rest of this paper is organized as follows: In Section 2, basic definitions and preliminaries are given. In Section 3, we investigate the existence of $(p, q)$ Bessel/frame measures. We show that every finite Borel measure $\nu$ is a $(p, q)$-Bessel measure for a finite measure $\mu$. In addition, we construct a large number of examples of measures which admit infinite discrete $(p, q)$-Bessel measures, by F-spectral measures and applying the Riesz-Thorin interpolation theorem. In general, for every spectral measure (B-spectral measure, or F-spectral measure respectively) $\mu$, there exists a discrete measure $\nu=\sum_{\lambda \in \Lambda_{\mu}} \delta_{\lambda}$ which is a Plancherel measure (Bessel measure or frame measure respectively) for $\mu$. Then the Riesz-Thorin interpolation theorem yields that $\nu$ is also a $(p, q)$-Bessel measure for $\mu$, where $1 \leq p \leq 2$ and $q$ is the conjugate exponent to $p$. Moreover, this shows that if $\mu$ is a spectral measure (B-spectral measure, or F-spectral measure), then the set $\left\{e_{\lambda}\right\}_{\lambda \in \Lambda_{\mu}}$ forms a $q$-Bessel sequence for $L^{p}(\mu)$. It is known $[13,19]$ that if a measure $\mu$ is an F-spectral measure, then it must be of pure type, i.e., $\mu$ is either discrete, absolutely continuous or singular continuous. Therefore, we consider such measures in constructing the examples. The interested reader can refer to $[3,6,7,9,13,16,18,19,20,21,23,24]$ to see examples and properties of spectral measures (B-spectral measures, or Fspectral measures) and related concepts. Besides discrete $(p, q)$-Bessel measures $\nu=\sum_{\lambda \in \Lambda_{\mu}} \delta_{\lambda}$ associated to spectral measures (B-spectral measures, or F-spectral measures) $\mu$, we prove that there exists an infinite absolutely continuous $(p, q)$-Bessel measure $\nu$ for some finite measures $\mu$ (see Proposition 3.12 and Example 4.1). We show that if $\nu$ is a $\left(p_{1}, q_{1}\right)$-Bessel/frame measure and $\left(p_{2}, q_{2}\right)$-Bessel/frame measure for $\mu$, where $1 \leq p_{1}, p_{2}<\infty$ and $q_{1}, q_{2}$ are the conjugate exponents to $p_{1}, p_{2}$, respectively, then $\nu$ is a $(p, q)$-Bessel measure for $\mu$ too, where $p_{1}<p<p_{2}$ and $q$ is the conjugate exponent to $p$. Consequently, if $\nu$ is a Bessel/frame measure for $\mu$, then it is a $(p, q)$-Bessel measure for $\mu$ too. In Proposition 3.10 we prove that there exists a measure $\mu$ which admits tight $(p, q)$-frame measures and $(p, q)$-Plancherel measures. Section 4 is devoted to investigating properties of $(p, q)$-Bessel/frame 
measures based on the results by Dutkay, Han, and Weber from [5].

\section{Preliminaries}

Definition 2.1. Let $t \in \mathbb{R}^{d}$. Denoted by $e_{t}$ the exponential function

$$
e_{t}(x)=e^{2 \pi i t \cdot x} \quad\left(x \in \mathbb{R}^{d}\right) .
$$

Definition 2.2. Let $H$ be a Hilbert space. A sequence $\left\{f_{i}\right\}_{i \in I}$ of elements in $H$ is called a Bessel sequence for $H$ if there exists a positive constant $B$ such that for all $f \in H$,

$$
\sum_{i \in I}\left|\left\langle f, f_{i}\right\rangle\right|^{2} \leq B\|f\|^{2} .
$$

Here $B$ is called the Bessel bound for the Bessel sequence $\left\{f_{i}\right\}_{i \in I}$.

The sequence $\left\{f_{i}\right\}_{i \in I}$ is called a frame for $H$, if there exist constants $A, B>0$ such that for all $f \in H$,

$$
A\|f\|^{2} \leq \sum_{i \in I}\left|\left\langle f, f_{i}\right\rangle\right|^{2} \leq B\|f\|^{2} .
$$

In this case, $A$ and $B$ are called frame bounds.

Frames are a natural generalization of orthonormal bases. It is easily seen from the lower bound that a frame is complete in $\mathrm{H}$, so every $f$ can be expressed using (infinite) linear combination of the elements $f_{i}$ in the frame [2].

Definition 2.3. Let $\mu$ be a compactly supported probability measure on $\mathbb{R}^{d}$ and $\Lambda$ be a countable set in $\mathbb{R}^{d}$, the set $E(\Lambda)=\left\{e_{\lambda}: \lambda \in \Lambda\right\}$ is called a Fourier frame for $L^{2}(\mu)$ if for all $f \in L^{2}(\mu)$,

$$
A\|f\|_{L^{2}(\mu)}^{2} \leq \sum_{\lambda \in \Lambda}\left|\left\langle f, e_{\lambda}\right\rangle_{L^{2}(\mu)}\right|^{2} \leq B\|f\|_{L^{2}(\mu)}^{2} .
$$

When $E(\Lambda)$ is an orthonormal basis (Bessel sequence, or frame) for $L^{2}(\mu)$, we say that $\mu$ is a spectral measure (B-spectral measure, or F-spectral measure respectively) and $\Lambda$ is called a spectrum (B-spectrum, or F-spectrum respectively) for $\mu$.

We give the following definition from [5], assuming that the given measure $\mu$ is a finite Borel measure on $\mathbb{R}^{d}$.

Definition 2.4. [[5]] Let $\mu$ be a finite Borel measure on $\mathbb{R}^{d}$. A Borel measure $\nu$ is called a Bessel measure for $\mu$, if there exists a positive constant $B$ such that for every $f \in L^{2}(\mu)$,

$$
\|\widehat{f d \mu}\|_{L^{2}(\nu)}^{2} \leq B\|f\|_{L^{2}(\mu)}^{2} .
$$

Here $B$ is called a (Bessel) bound for $\nu$. 
The measure $\nu$ is called a frame measure for $\mu$ if there exist positive constants $A, B$ such that for every $f \in L^{2}(\mu)$,

$$
A\|f\|_{L^{2}(\mu)}^{2} \leq\|\widehat{f d \mu}\|_{L^{2}(\nu)}^{2} \leq B\|f\|_{L^{2}(\mu)}^{2} .
$$

In this case, $A$ and $B$ are called (frame) bounds for $\nu$. The measure $\nu$ is called a tight frame measure if $A=B$ and Plancherel measure if $A=B=1$ (see also [8]).

The set of all Bessel measures for $\mu$ with fixed bound $B$ is denoted by $\mathcal{B}_{B}(\mu)$ and the set of all frame measures for $\mu$ with fixed bounds $A, B$ is denoted by $\mathcal{F}_{A, B}(\mu)$.

Remark 2.1. A compactly supported probability measure $\mu$ is an F-spectral measure if and only if there exists a countable set $\Lambda$ in $\mathbb{R}^{d}$ such that $\nu=\sum_{\lambda \in \Lambda} \delta_{\lambda}$ is a frame measure for $\mu$.

Definition 2.5. A finite set of contraction mappings $\left\{\tau_{i}\right\}_{i=1}^{n}$ on a complete metric space is called an iterated function system (IFS). Hutchinson [15] proved that, for the metric space $\mathbb{R}^{d}$, there exists a unique compact subset $X$ of $\mathbb{R}^{d}$, which satisfies $X=\bigcup_{i=1}^{n} \tau_{i}(X)$. Moreover, if the IFS is associated with a set of probability weights $\left\{\rho_{i}\right\}_{i=1}^{n}$ (i.e., $0<\rho_{i}<1, \sum_{i=1}^{n} \rho_{i}=1$ ), then there exists a unique Borel probability measure $\mu$ supported on $X$ such that $\mu=\sum_{i=1}^{n} \rho_{i}\left(\mu \circ \tau_{i}^{-1}\right)$. The corresponding $X$ and $\mu$ are called the attractor and the invariant measure of the IFS, respectively. It is well known that the invariant measure is either absolutely continuous or singular continuous with respect to Lebesgue measure. In an affine IFS each $\tau_{i}$ is affine and represented by a matrix. If $R$ is a $d \times d$ expanding integer matrix (i.e., all eigenvalues $\lambda$ satisfy $|\lambda|>1$ ), and $\mathcal{A} \subset \mathbb{Z}^{d}$, with $\# \mathcal{A}=: N \geq 2$, then the following set (associated with a set of probability weights) is an affine iterated function system.

$$
\tau_{a}(x)=R^{-1}(x+a) \quad\left(x \in \mathbb{R}^{d}, a \in \mathcal{A}\right) .
$$

Since $R$ is expanding, the maps $\tau_{a}$ are contractions (in an appropriate metric equivalent to the Euclidean one). In some cases, the invariant measure $\mu_{\mathcal{A}}$ is a fractal measure (see [3]). For example singular continuous invariant measures supported on Cantor type sets are fractal measures (see $[15,14]$ ).

Definition 2.6. [[22]](Semi-inner product spaces)

Let $X$ be a vector space over the filed $F$ of complex (real) numbers. If a function $[\cdot, \cdot]: X \times X \rightarrow F$ satisfies the following properties:

1. $[x+y, z]=[x, z]+[y, z], \quad$ for $x, y, z \in X$;

2. $[\lambda x, y]=\lambda[x, y]$, for $\lambda \in F$ and $x, y \in X$;

3. $[x, x]>0$, for $x \neq 0$;

4. $|[x, y]|^{2} \leq[x, x][y, y]$, 
then $[\cdot, \cdot]$ is called a semi-inner product and the pair $(X,[\cdot, \cdot])$ is called a semi-inner product space. It is easy to observe that $\|x\|=[x, x]^{\frac{1}{2}}$ is a norm on $X$. So every semi-inner product space is a normed linear space. On the other hand, one can generate a semi-inner product in a normed linear space, in infinitely many different ways.

As a matter of fact, semi-inner products provide the possibility of carrying over Hilbert space type arguments to Banach spaces.

Every Banach space has a semi-inner product that is compatible. For example consider the Banach function space $L^{p}(X, \mu), p \geq 1$, a compatible semi-inner product on this space is defined by (see [12])

$$
[f, g]_{L^{p}(\mu)}:=\frac{1}{\|g\|_{L^{p}(\mu)}^{p-2}} \int_{X} f(x)|g(x)|^{p-1} \overline{\operatorname{sgn}(g(x))} d \mu(x),
$$

for every $f, g \in L^{p}(X, \mu)$ with $\|g\|_{L^{p}(\mu)} \neq 0$, and $[f, g]_{L^{p}(\mu)}=0$ for $\|g\|_{L^{p}(\mu)}=0$.

To construct frames in a Hilbert space $H$ the sequence space $l^{2}$ is required. Similarly, to construct frames in a Banach space $X$ one needs a Banach space of scaler valued sequences $X_{d}$ (in fact a BK-space $X_{d}$, see [1] and the references therein). According to Zhang and Zhang [26] frames in Banach spaces can be defined via a compatible semi-inner product in the following way:

Definition 2.7. Let $X$ be a Banach space with a compatible semi-inner product $[\cdot, \cdot]$ and norm $\|\cdot\|_{X}$. Let $X_{d}$ be an associated BK-space with norm $\|\cdot\|_{X_{d}}$. A sequence of elements $\left\{f_{i}\right\}_{i \in I} \subseteq X$ is called an $X_{d}$-frame for $X$ if $\left\{\left[f, f_{i}\right]\right\}_{i \in I} \in X_{d}$ for all $f \in X$, and there exist constants $A, B>0$ such that for every $f \in X$,

$$
A\|f\|_{X} \leq\left\|\left\{\left[f, f_{i}\right]\right\}_{i \in I}\right\|_{X_{d}} \leq B\|f\|_{X} .
$$

See also [25]

Based on Definition 2.7, we present the next definition. We consider the function space $L^{p}(\mu)$ and the sequence space $l^{q}(I)$ (where $p>1$ and $q$ is the conjugate exponent to $p$ ) as the Banach space and the BK- space, respectively.

Definition 2.8. Suppose that $1<p, q<\infty$ and $1 / p+1 / q=1$. Let $\mu$ be a finite Borel measure on $\mathbb{R}^{d}$ and let $[\cdot, \cdot]$ be the compatible semi-inner product on $L^{p}(\mu)$ as defined above. We say that a sequence $\left\{f_{i}\right\}_{i \in I}$ is a $q$-Bessel sequence for $L^{p}(\mu)$ if there exists a constant $B>0$ such that for every $f \in L^{p}(\mu)$,

$$
\sum_{i \in I}\left|\left[f, f_{i}\right]_{L^{p}(\mu)}\right|^{q} \leq B\|f\|_{L^{p}(\mu)}^{q} .
$$

We call B a (q-Bessel) bound. 
We say the sequence $\left\{f_{i}\right\}_{i \in I}$ is a $q$-frame for $L^{p}(\mu)$ if there exist constants $A, B>0$ such that for every $f \in L^{p}(\mu)$,

$$
A\|f\|_{L^{p}(\mu)}^{q} \leq \sum_{i \in I}\left|\left[f, f_{i}\right]_{L^{p}(\mu)}\right|^{q} \leq B\|f\|_{L^{p}(\mu)}^{q} .
$$

We call $A, B$ (q-frame) bounds. We call the sequence $\left\{f_{i}\right\}_{i \in I}$ a tight $q$-frame if $A=B$ and Parseval $q$-frame if $A=B=1$.

We extend the notions of Bessel and frame measures as follows.

Definition 2.9. Suppose that $1 \leq p<\infty, 1<q \leq \infty$ and $1 / p+1 / q=1$. Let $\mu$ be a finite Borel measure on $\mathbb{R}^{d}$, and let $[\cdot, \cdot]$ be the compatible semi-inner product on $L^{p}(\mu)$ as defined above. We say that a Borel measure $\nu$ is a $(p, q)$-Bessel measure for $\mu$, if there exists a constant $B>0$ such that for every $f \in L^{p}(\mu)$,

$$
\int_{\mathbb{R}^{d}}\left|\left[f, e_{t}\right]_{L^{p}(\mu)}\right|^{q} d \nu(t) \leq B\|f\|_{L^{p}(\mu)}^{q} \quad(p \neq 1, q \neq \infty)
$$

and

We call $B$ a $((p, q)$-Bessel $)$ bound for $\nu$.

$$
\|\widehat{f d \mu}\|_{\infty} \leq B\|f\|_{L^{1}(\mu)} \quad(p=1, q=\infty) .
$$

We say the Borel measure $\nu$ is a $(p, q)$-frame measure for $\mu$, if there exist constants $A, B>0$ such that for every $f \in L^{p}(\mu)$,

$$
A\|f\|_{L^{p}(\mu)}^{q} \leq \int_{\mathbb{R}^{d}}\left|\left[f, e_{t}\right]_{L^{p}(\mu)}\right|^{q} d \nu(t) \leq B\|f\|_{L^{p}(\mu)}^{q} \quad(p \neq 1, q \neq \infty)
$$

and

$$
A\|f\|_{L^{1}(\mu)} \leq\|\widehat{f d \mu}\|_{\infty} \leq B\|f\|_{L^{1}(\mu)} \quad(p=1, q=\infty) .
$$

We call $A, B((p, q)$-frame) bounds for $\nu$. If $A=B$, we call the measure $\nu$ a tight $(p, q)$-frame measure and if $A=B=1$, we call it a $(p, q)$-Plancherel measure.

We denote the set of all $(p, q)$-Bessel measures for $\mu$ with fixed bound $B$ by $\mathcal{B}_{B}(\mu)_{p, q}$ and the set of all $(p, q)$-frame measures for $\mu$ with fixed bounds $A, B$ by $\mathcal{F}_{A, B}(\mu)_{p, q}$.

Remark 2.2. Since $\left[f, e_{t}\right]_{L^{p}(\mu)}=\int_{R^{d}} f(x) e^{-2 \pi i t \cdot x} d \mu(x)=\widehat{f d \mu}(t)$ for any $f \in L^{p}(\mu)$ and $t \in \mathbb{R}^{d}$, we can also write $\widehat{f d \mu}(t)$ instead of $\left[f, e_{t}\right]_{L^{p}(\mu)}$. If there exists a $(p, q)$-Bessel/frame measure $\nu$ for $\mu$, then the function $T_{\nu}: L^{p}(\mu) \rightarrow L^{q}(\nu)$ defined by $T_{\nu} f=\widehat{f d \mu}$ is linear and bounded. For $p=1, q=\infty$, every $\sigma$-finite measure $\nu$ on $\mathbb{R}^{d}$ is a $(1, \infty)$-Bessel measure for $\mu$, since we always have $\|\widehat{f d \mu}\|_{\infty} \leq\|f\|_{L^{1}(\mu)}$. More precisely, $\nu \in \mathcal{B}_{1}(\mu)_{(1, \infty)}$.

Theorem 2.1. [10] (Riesz-Thorin interpolation theorem) Let $1 \leq p_{0}, p_{1}, q_{0}, q_{1} \leq$ $\infty$, where $p_{0} \neq p_{1}$ and $q_{0} \neq q_{1}$, and let $T$ be a linear operator. Suppose that for some measure spaces $(Y, \nu),(X, \mu), T: L^{p_{0}}(X, \mu) \rightarrow L^{q_{0}}(Y, \nu)$ is bounded with norm $C_{0}$, and $T: L^{p_{1}}(X, \mu) \rightarrow L^{q_{1}}(Y, \nu)$ is bounded with norm $C_{1}$. Then for all $\theta \in(0,1)$ and $p, q$ defined by $1 / p=(1-\theta) / p_{0}+\theta / p_{1} ; 1 / q=(1-\theta) / q_{0}+\theta / q_{1}$, there exists $a$ constant $C$ such that $C \leq C_{0}^{(1-\theta)} C_{1}^{\theta}$ and $T: L^{p}(X, \mu) \rightarrow L^{q}(Y, \nu)$ is bounded with norm $C$. 


\section{Existence and Examples}

In this section, we will investigate the existence of $(p, q)$-Bessel and $(p, q)$-frame measures and also the existence of $q$-Bessel sequences and $q$-frames. In addition, we will construct the examples of measures which admit $(p, q)$-Bessel measures.

Proposition 3.1. Suppose that $1<p, q<\infty$ and $1 / p+1 / q=1$. Let $\mu$ be a finite Borel measure. Then every finite Borel measure $\nu$ is a $(p, q)$-Bessel measure for $\mu$.

Proof. Take $f \in L^{p}(\mu)$ and $t \in \mathbb{R}^{d}$. Then by applying Holder's inequality

$$
\left|\left[f, e_{t}\right]_{L^{p}(\mu)}\right| \leq \int_{\mathbb{R}^{d}}\left|f(x) e^{-2 \pi i t \cdot x}\right| d \mu(x) \leq\left(\mu\left(\mathbb{R}^{d}\right)\right)^{\frac{1}{q}}\|f\|_{L^{p}(\mu)} .
$$

Thus,

$$
\int_{\mathbb{R}^{d}}\left|\left[f, e_{t}\right]_{L^{p}(\mu)}\right|^{q} d \nu(t) \leq \mu\left(\mathbb{R}^{d}\right) \nu\left(\mathbb{R}^{d}\right)\|f\|_{L^{p}(\mu)}^{q} .
$$

Therefore $\nu \in \mathcal{B}_{\mu\left(\mathbb{R}^{d}\right) \nu\left(\mathbb{R}^{d}\right)}(\mu)_{(p, q)}$. For $p=1, q=\infty$, as we mentioned in Remark 2.2 $\nu \in \mathcal{B}_{1}(\mu)_{(1, \infty)}$.

Proposition 3.2. Suppose that $1<p, q<\infty$ and $1 / p+1 / q=1$. Let $\Lambda \subset \mathbb{R}^{d}$, $\# \Lambda<\infty$ and let $\mu$ be a finite Borel measure. Then the finite sequence $\left\{e_{\lambda}\right\}_{\lambda \in \Lambda}$ is a q-Bessel sequence for $L^{p}(\mu)$.

Proof. Consider the finite discrete measure $\nu=\sum_{\lambda \in \Lambda} \delta_{\lambda}$. Since

$$
\sum_{\lambda \in \Lambda}\left|\left[f, e_{\lambda}\right]_{L^{p}(\mu)}\right|^{q}=\int_{\mathbb{R}^{d}}\left|\left[f, e_{t}\right]_{L^{p}(\mu)}\right|^{q} d \nu(t),
$$

then the assertion follows from Proposition 3.1.

Remark 3.1. Proposition 3.1 shows that the Bessel bound may change for different measures $\nu$. So if we consider Borel probability measures $\nu$, then we have a fixed Bessel bound $\mu\left(\mathbb{R}^{d}\right)$ for all $\nu$. Moreover, this Bessel bound does not depend on $p, q$, i.e., for every probability measure $\nu$ we have $\nu \in \mathcal{B}_{\mu\left(\mathbb{R}^{d}\right)}(\mu)_{(p, q)}$, where $1<p<\infty$ and $q$ is the conjugate exponent to $p$. In addition, we obtain from Proposition 3.1 that for all conjugate exponents $p, q>1$ the set $\mathcal{B}_{\mu\left(\mathbb{R}^{d}\right)}(\mu)_{p, q}$ is infinite, since there are infinitely many probability measures $\nu$ (such as every measure $\nu=\frac{1}{\lambda(S)} \chi_{S} d \lambda$ where $S \subset \mathbb{R}^{d}$ with the finite Lebesgue measure $\lambda(S)$, every finite discrete measure $\nu=\frac{1}{n} \sum_{a=1}^{n} \delta_{a}$ where $\delta_{a}$ denotes the Dirac measure at the point $a$, every invariant measure obtained from an iterated function system, and others).

Proposition 3.3. Suppose that $1<p, q<\infty$ and $1 / p+1 / q=1$. Let $\nu$ be a finite Borel measure. Then $\nu$ is a $(p, q)$-Bessel measure for every finite Borel measure $\mu$. In addition, $\nu \in \mathcal{B}_{\nu\left(\mathbb{R}^{d}\right)}(\mu)_{(p, q)}$ for all probability measures $\mu$.

Proof. See the proof of Proposition 3.1. 
Corollary 3.1. Suppose that $1<p, q<\infty$ and $1 / p+1 / q=1$. A finite Borel measure $\nu$ is a $(p, q)$-Bessel measure for a finite Borel measure $\mu$, if and only if $\mu$ is a $(p, q)$-Bessel measure for $\nu$. In particular, every finite Borel measure $\mu$ is a $(p, q)$-Bessel measure to itself.

Proof. The statements are direct consequences of Propositions 3.1 and 3.3.

Lemma 3.1. Suppose that $1<p, q<\infty$ and $1 / p+1 / q=1$. Let $\mu$ be a finite Borel measure. Then the following assertions hold.

(i) If there exists a countable set $\Lambda$ in $\mathbb{R}^{d}$ such that $\left\{e_{\lambda}\right\}_{\lambda \in \Lambda}$ is a $q$-frame for $L^{p}(\mu)$, then $\nu=\sum_{\lambda \in \Lambda} \delta_{\lambda}$ is a $(p, q)$-frame measure for $\mu$.

(ii) If $\nu$ is purely atomic, i.e., $\nu=\sum_{\lambda \in \Lambda} d_{\lambda} \delta_{\lambda}$, and a $(p, q)$-frame measure for the probability measure $\mu$, then $\left\{\sqrt[q]{d_{\lambda}} e_{\lambda}\right\}_{\lambda \in \Lambda}$ is a q-frame for $L^{p}(\mu)$.

Proof. (i) Let $\nu=\sum_{\lambda \in \Lambda} \delta_{\lambda}$. Then for all $f \in L^{p}(\mu)$,

$$
\sum_{\lambda \in \Lambda}\left|\left[f, e_{\lambda}\right]_{L^{p}(\mu)}\right|^{q}=\int_{\mathbb{R}^{d}}\left|\left[f, e_{t}\right]_{L^{p}(\mu)}\right|^{q} d \nu(t) .
$$

(ii) Since for all $f \in L^{p}(\mu)$,

$$
\int_{\mathbb{R}^{d}}\left|\left[f, e_{t}\right]_{L^{p}(\mu)}\right|^{q} d \nu(t)=\sum_{\lambda \in \Lambda} d_{\lambda}\left|\left[f, e_{\lambda}\right]_{L^{p}(\mu)}\right|^{q}=\sum_{\lambda \in \Lambda}\left|\left[f, \sqrt[q]{d_{\lambda}} e_{\lambda}\right]\right|^{q} .
$$

Example 3.1. Suppose that $1 \leq p \leq 2$ and $q$ is the conjugate exponent to $p$. If $f \in$ $L^{p}\left([0,1]^{d}\right)$, then from the Hausdorff-Young inequality we have $\hat{f} \in l^{q}\left(\mathbb{Z}^{d}\right)$ and $\|\hat{f}\|_{q} \leq$ $\|f\|_{p}$. Therefore, the measure $\nu=\sum_{t \in \mathbb{Z}^{d}} \delta_{t}$ is a $(p, q)$-Bessel measure for $\mu=\chi_{\left\{[0,1]^{d}\right\}} d x$. Besides, $\left\{e_{t}\right\}_{t \in \mathbb{Z}^{d}}$ is a $q$-Bessel sequence for $L^{p}(\mu)$, since $\sum_{t \in \mathbb{Z}^{d}}\left|\left[f, e_{t}\right]_{L^{p}(\mu)}\right|^{q} \leq\|f\|_{p}^{q}$, where $1<p \leq 2$ and $q$ is the conjugate exponent to $p$.

Proposition 3.4. Suppose $1<p \leq 2$ and $q$ is the conjugate exponent to $p$. Let $\mu=\chi_{\left\{[0,1]^{d}\right\}} d x$ and let $0<a \leq \phi(x) \leq b<\infty$ on $[0,1]^{d}$. If $\phi_{t}(x):=\phi(x)$ for all $t \in \mathbb{Z}^{d}$, then $\left\{\phi_{t} e_{t}\right\}_{t \in \mathbb{Z}^{d}}$ is a $q$-Bessel sequence for $L^{p}(\mu)$.

Proof. Take $f \in L^{p}(\mu)$. We have $\frac{1}{\|\phi\|_{p}^{p-2}} \phi^{p-1} f \in L^{p}(\mu)$, since

$$
\int_{\mathbb{R}^{d}}|f(x)|^{p}\left|\frac{\phi^{p-1}(x)}{\|\phi\|_{p}^{p-2}}\right|^{p} d \mu(x) \leq \frac{b^{(p-1) p}}{a^{(p-2) p}} \int_{\mathbb{R}^{d}}|f(x)|^{p} d \mu(x)<\infty .
$$

Hence by Example 3.1,

$$
\begin{aligned}
\sum_{t \in \mathbb{Z}^{d}}\left|\left[f, \phi_{t} e_{t}\right]_{L^{p}(\mu)}\right|^{q} & =\left.\left.\sum_{t \in \mathbb{Z}^{d}}\left|\frac{1}{\|\phi\|_{p}^{p-2}} \int_{\mathbb{R}^{d}} f(x)\right| \phi(x) e_{t}(x)\right|^{p-1} e_{-t}(x) d \mu(x)\right|^{q} \\
& \leq\left.\left.\left|\int_{\mathbb{R}^{d}}\right| f(x)\right|^{p}\left|\frac{\phi^{p-1}(x)}{\|\phi\|_{p}^{p-2}}\right|^{p} d \mu(x)\right|^{q / p} \leq \frac{b^{p}}{a^{p-q}}\|f\|_{p}^{q} .
\end{aligned}
$$


Corollary 3.2. Suppose that $1<p, q<\infty$ and $1 / p+1 / q=1$. Let $\mu$ be $a$ probability measure. Let $0<a \leq \phi(x) \leq b<\infty$ on $\operatorname{supp} \mu$ and $\phi_{i}(x):=\phi(x)$ for all $i \in I$. If $\left\{f_{i}\right\}_{i \in I}$ is a q-frame for $L^{p}(\mu)$, then $\left\{\phi_{i} f_{i}\right\}_{i \in I}$ is also a $q$-frame for $L^{p}(\mu)$ and for every $f \in L^{p}(\mu)$,

$$
\frac{a^{p}}{b^{p-q}} A\|f\|_{L^{p}(\mu)}^{q} \leq\left\|\left\{\left[f, \phi_{i} f_{i}\right]_{L^{p}(\mu)}\right\}_{i \in I}\right\|^{q} \leq \frac{b^{p}}{a^{p-q}} B\|f\|_{L^{p}(\mu)}^{q} .
$$

Remark 3.2. Example 3.1 cannot be extended to the case $p>2$, since there exist continuous functions $f$ such that $\sum_{n \in \mathbb{Z}}\left|\left[f, e_{n}\right]_{L^{p}(\mu)}\right|^{2-\epsilon}=\infty$ for all $\epsilon>0$. Therefore, $\nu=\sum_{n \in \mathbb{Z}} \delta_{n}$ is not a $(p, q)$-Bessel measure for $\mu=\chi_{[0,1]} d x$ where $p>2$ and also $\left\{e_{n}\right\}_{n \in \mathbb{Z}}$ is not a $q$-Bessel sequence for $L^{p}(\mu)$. As an example take $f(x)=\sum_{n=2}^{\infty} \frac{e^{i n \log n}}{n^{1 / 2}(\log n)^{2}} e^{i n x}$ (see $[17])$.

Proposition 3.5. Suppose that $1<p, q<\infty$ and $1 / p+1 / q=1$. Let $\mu$ be $a$ compactly supported Borel probability measure. Consider two subsets of $\mathbb{R}^{d}, \Lambda=$ $\left\{\lambda_{n}: n \in \mathbb{N}\right\}$ and $\Omega=\left\{\omega_{n}: n \in \mathbb{N}\right\}$ with the property that there exists a positive constant $C$ such that $\left|\lambda_{n}-\omega_{n}\right| \leq C$ for $n \in \mathbb{N}$.

(i) If $\left\{e_{\lambda_{n}}\right\}_{n \in \mathbb{N}}$ is a q-Bessel sequence for $L^{p}(\mu)$, then $\left\{e_{\omega_{n}}\right\}_{n \in \mathbb{N}}$ is a q-Bessel sequence too.

(ii) If $\left\{e_{\lambda_{n}}\right\}_{n \in \mathbb{N}}$ is a $q$-frame for $L^{p}(\mu)$, then there exists a $\delta>0$ such that if $C \leq \delta$ then $\left\{e_{\omega_{n}}\right\}_{n \in \mathbb{N}}$ is a q-frame too (see [3]).

Proof. We need only consider the case, when all $\omega_{n}=\left(\left(\omega_{n}\right)_{1}, \ldots,\left(\omega_{n}\right)_{d}\right)$ differ from $\lambda_{n}=\left(\left(\lambda_{n}\right)_{1}, \ldots,\left(\lambda_{n}\right)_{d}\right)$ just on the first component, then the assertion follows by induction on the number of components.

Let $\operatorname{supp} \mu \subseteq[-M, M]^{d}$ for some $M>0$. Let $f \in L^{p}(\mu)$ and $x \in \mathbb{R}^{d}$. The function $\widehat{f d \mu}$ is analytic in each variable $t_{1}, \ldots, t_{d}$. Moreover, for every $t \in \mathbb{R}^{d}$

$$
\frac{\partial^{k} \widehat{f d \mu}}{\partial t_{1}^{k}}(t)=\int f(x)\left(-2 \pi i x_{1}\right)^{k} e^{-2 \pi i t \cdot x} d \mu(x)=\left[\left(-2 \pi i x_{1}\right)^{k} f, e_{t}\right]_{L^{p}(\mu)} .
$$

Writing the Taylor expansion at $\left(\lambda_{n}\right)_{1}$ in the first variable and using Holder's inequality, for all $n \in \mathbb{N}$,

$$
\begin{aligned}
\left|\widehat{f d \mu}\left(\omega_{n}\right)-\widehat{f d \mu}\left(\lambda_{n}\right)\right|^{q} & =\left|\sum_{k=1}^{\infty} \frac{\frac{\partial^{k} \widehat{f d \mu}}{\partial t_{1}^{k}}\left(\lambda_{n}\right)}{k !}\left(\left(\omega_{n}\right)_{1}-\left(\lambda_{n}\right)_{1}\right)^{k}\right|^{q} \\
& \leq \sum_{k=1}^{\infty} \frac{\left|\frac{\partial^{k} \widehat{f d \mu}}{\partial t_{1}^{k}}\left(\lambda_{n}\right)\right|^{q}}{k !} \cdot\left(\sum_{k=1}^{\infty} \frac{\left|\left(\omega_{n}\right)_{1}-\left(\lambda_{n}\right)_{1}\right|^{p k}}{k !}\right)^{q / p} \\
& \leq \sum_{k=1}^{\infty} \frac{\left|\frac{\partial^{k} \widehat{f d \mu}}{\partial t_{1}^{k}}\left(\lambda_{n}\right)\right|^{q}}{k !} \cdot\left(\sum_{k=1}^{\infty} \frac{C^{p k}}{k !}\right)^{q-1} \\
& =\sum_{k=1}^{\infty} \frac{\left|\frac{\partial^{k} \widehat{f d \mu}}{\partial t_{1}^{k}}\left(\lambda_{n}\right)\right|^{q}}{k !} \cdot\left(e^{C^{p}}-1\right)^{q-1} .
\end{aligned}
$$


Considering the $q$-Bessel sequence $\left\{e_{\lambda_{n}}\right\}_{n \in \mathbb{N}}$ with a bound $B$, we obtain

$$
\begin{aligned}
\sum_{n \in \mathbb{N}}\left|\frac{\partial^{k} \widehat{f d \mu}}{\partial t_{1}^{k}}\left(\lambda_{n}\right)\right|^{q} & =\sum_{n \in \mathbb{N}}\left|\left[\left(-2 \pi i x_{1}\right)^{k} f, e_{\lambda_{n}}\right]_{L^{p}(\mu)}\right|^{q} \leq B\left\|\left(-2 \pi i x_{1}\right)^{k} f\right\|_{L^{p}(\mu)}^{q} \\
& \leq B(2 \pi M)^{q k}\|f\|_{L^{p}(\mu)}^{q} .
\end{aligned}
$$

Then

$$
\begin{aligned}
\sum_{n \in \mathbb{N}}\left|\widehat{f d \mu}\left(\omega_{n}\right)-\widehat{f d \mu}\left(\lambda_{n}\right)\right|^{q} & \leq B\left(e^{C^{p}}-1\right)^{q-1}\|f\|_{L^{p}(\mu)}^{q} \sum_{k=1}^{\infty} \frac{(2 \pi M)^{q k}}{k !} \\
& =B\left(e^{C^{p}}-1\right)^{q-1}\left(e^{(2 \pi M)^{q}}-1\right)\|f\|_{L^{p}(\mu)}^{q} .
\end{aligned}
$$

Hence by Minkowski's inequality,

$$
\begin{aligned}
\left(\sum_{n \in \mathbb{N}}\left|\widehat{f d \mu}\left(\omega_{n}\right)\right|^{q}\right)^{1 / q} & \leq\left(\sum_{n \in \mathbb{N}}\left|\widehat{f d \mu}\left(\lambda_{n}\right)\right|^{q}\right)^{1 / q}+\left(\sum_{n \in \mathbb{N}}\left|\widehat{f d \mu}\left(\omega_{n}\right)-\widehat{f d \mu}\left(\lambda_{n}\right)\right|^{q}\right)^{1 / q} \\
& \leq\left(B^{1 / q}+\left(B\left(e^{C^{p}}-1\right)^{q-1}\left(e^{(2 \pi M)^{q}}-1\right)\right)^{1 / q}\right)\|f\|_{L^{p}(\mu)},
\end{aligned}
$$

and this implies that $\left\{e_{\omega_{n}}\right\}_{n \in \mathbb{N}}$ is a $q$-Bessel sequence for $L^{p}(\mu)$.

To show that $\left\{e_{\omega_{n}}\right\}_{n \in \mathbb{N}}$ is also a $q$-frame for $L^{p}(\mu)$, let $A$ be a lower bound for $\left\{e_{\lambda_{n}}\right\}_{n \in \mathbb{N}}$. Take $\delta>0$ small enough such that for $0<C \leq \delta$,

$$
A^{1 / q}-\left(B\left(e^{C^{p}}-1\right)^{q-1}\left(e^{(2 \pi M)^{q}}-1\right)\right)^{1 / q}>0 .
$$

Then, by Minkowski's inequality,

$$
\begin{aligned}
\left(\sum_{n \in \mathbb{N}}\left|\widehat{f d \mu}\left(\omega_{n}\right)\right|^{q}\right)^{1 / q} & \geq\left(\sum_{n \in \mathbb{N}}\left|\widehat{f d \mu}\left(\lambda_{n}\right)\right|^{q}\right)^{1 / q}-\left(\sum_{n \in \mathbb{N}}\left|\widehat{f d \mu}\left(\omega_{n}\right)-\widehat{f d \mu}\left(\lambda_{n}\right)\right|^{q}\right)^{1 / q} \\
& \geq\left(A^{1 / q}-\left(B\left(e^{C^{p}}-1\right)^{q-1}\left(e^{(2 \pi M)^{q}}-1\right)\right)^{1 / q}\right)\|f\|_{L^{p}(\mu)} .
\end{aligned}
$$

Thus the assertion follows.

Proposition 3.6. Suppose that $1 \leq p_{0}, p_{1}<\infty$ and $q_{0}, q_{1}$ are the conjugate exponents to $p_{0}, p_{1}$ respectively. If $\nu$ is a $\left(p_{0}, q_{0}\right)$-Bessel measure and a $\left(p_{1}, q_{1}\right)$-Bessel measure for $\mu$, then $\nu$ is also a $(p, q)$-Bessel measure for $\mu$, where $p_{0}<p<p_{1}$ and $q$ is the conjugate exponent to $p$.

Proof. If $\nu$ is a $\left(p_{0}, q_{0}\right)$-Bessel measure for $\mu$ with bound $C$ and also a $\left(p_{1}, q_{1}\right)$-Bessel measure with bound $D$, we have

$$
\forall f \in L^{p_{0}}(\mu) \quad\|\widehat{f d \mu}\|_{L^{q_{0}}(\nu)}^{q_{0}} \leq C\|f\|_{L^{p_{0}}(\mu)}^{q_{0}},
$$


and

$$
\forall f \in L^{p_{1}}(\mu) \quad\|\widehat{f d \mu}\|_{L^{q_{1}}(\nu)}^{q_{1^{\prime}}} \leq D\|f\|_{L^{p_{1}}(\mu)}^{q_{1}} .
$$

Now if $1 / p=(1-\theta) / p_{0}+\theta / p_{1} ; 1 / q=(1-\theta) / q_{0}+\theta / q_{1}$, where $0<\theta<1$ (i.e., $p_{0}<p<p_{1}$ and $\left.1 / p+1 / q=1\right)$, then the Riesz-Thorin interpolation theorem yields

$$
\forall f \in L^{p}(\mu) \quad\|\widehat{f d \mu}\|_{L^{q}(\nu)}^{q} \leq B^{q}\|f\|_{L^{p}(\mu)}^{q} .
$$

where $B \leq C^{\frac{1}{q_{0}}(1-\theta)} D^{\frac{1}{q_{1}} \theta}$ (Considering the fact that if $p_{0}=1$ and $q_{0}=\infty$, then $C^{\frac{1}{q_{0}}}$ changes to $C$, and if $p_{1}=1$ and $q_{1}=\infty$, then $D^{\frac{1}{q_{1}}}$ changes to $D$ ). Hence $\nu$ is a $(p, q)$-Bessel measure for $\mu$, where $p_{0}<p<p_{1}$ and $q$ is the conjugate exponent to $p$.

Corollary 3.3. If $\nu$ is a Bessel/frame measure for $\mu$, then $\nu$ is also a $(p, q)$-Bessel measure for $\mu$, where $1 \leq p \leq 2$ and $q$ is the conjugate exponent to $p$.

Proof. Let $p_{0}=1, q_{0}=\infty, p_{1}=2, q_{1}=2$ in the assumption of Proposition 3.6, then the conclusion follows.

Proposition 3.7. If $\nu \in \mathcal{F}_{A, B}(\mu)$, then for any constant $\alpha>0, \nu$ is a frame measure for $\alpha \mu$. More precisely $\nu \in \mathcal{F}_{\alpha A, \alpha B}(\alpha \mu)$.

Proof. Since $\nu \in \mathcal{F}_{A, B}(\mu)$ for all $f \in L^{2}(\mu)$,

$$
A\|f\|_{L^{2}(\mu)}^{2} \leq\|\widehat{f d \mu}\|_{L^{2}(\nu)}^{2} \leq B\|f\|_{L^{2}(\mu)}^{2},
$$

and we have

$$
\|\widehat{\alpha f d \mu}\|_{L^{2}(\nu)}^{2}=\int_{\mathbb{R}^{d}}\left|\int_{\mathbb{R}^{d}} f(x) e_{-t}(x) d \alpha \mu(x)\right|^{2} d \nu(t)=\|\widehat{f d \alpha \mu}\|_{L^{2}(\nu)}^{2} .
$$

Since $\alpha f \in L^{2}(\mu)$,

$$
A\|\alpha f\|_{L^{2}(\mu)}^{2} \leq\|\widehat{\alpha f d \mu}\|_{L^{2}(\nu)}^{2} \leq B\|\alpha f\|_{L^{2}(\mu)}^{2} \quad \text { for all } f \in L^{2}(\mu) .
$$

Therefore,

$$
\alpha A\|f\|_{L^{2}(\alpha \mu)}^{2} \leq\|\widehat{f d \alpha \mu}\|_{L^{2}(\nu)}^{2} \leq \alpha B\|f\|_{L^{2}(\alpha \mu)}^{2} \quad \text { for all } f \in L^{2}(\alpha \mu) .
$$

Hence $\nu \in \mathcal{F}_{\alpha A, \alpha B}(\alpha \mu)$.

Theorem 3.1. [23] There exists positive constants c, $C$ such that for every set $S \subset$ $\mathbb{R}^{d}$ of finite measure, there is a discrete set $\Lambda \subset \mathbb{R}^{d}$ such that $E(\Lambda)$ is a frame for $L^{2}(S)$ with frame bounds $c|S|$ and $C|S|$, where $|S|$ denotes the measure of $S$.

Theorem 3.2. Let $S$ be a subset (not necessarily bounded) of $\mathbb{R}^{d}$ with finite Lebesgue measure $|S|$. Then the probability measure $\mu=\frac{1}{|S|} \chi_{S} d x$ has an infinite discrete $(p, q)$-Bessel measure $\nu$, where $1 \leq p \leq 2$ and $q$ is the conjugate exponent to $p$. 
Proof. By Theorem 3.1, there are positive constants $c, C$ such that for every set $S \subset \mathbb{R}^{d}$ of finite Lebesgue measure $|S|$, there is a discrete set $\Lambda \subset \mathbb{R}^{d}$ such that $E(\Lambda)$ is a frame for $L^{2}(S)$ with frame bounds $c|S|$ and $C|S|$. Then by considering the upper bound of the frame, we have

$$
\sum_{\lambda \in \Lambda}\left|\left\langle f, e_{\lambda}\right\rangle\right|^{2} \leq C|S|\|f\|_{L^{2}(S)}^{2} \quad \text { for all } f \in L^{2}(S) .
$$

Let $\mu=\frac{1}{|S|} \chi_{S} d x$, and then by Proposition 3.7,

$$
\sum_{\lambda \in \Lambda}\left|\left\langle f, e_{\lambda}\right\rangle\right|^{2} \leq C\|f\|_{L^{2}(\mu)}^{2} \quad \text { for all } f \in L^{2}(\mu) .
$$

In addition, $\left\|\left\{\left[f, e_{\lambda}\right]_{L^{1}(\mu)}\right\}_{\lambda \in \Lambda}\right\|_{\infty} \leq\|f\|_{L^{1}(\mu)}$, for every $f$ in $L^{1}(\mu)$. Now if $1 / p=$ $1-\theta / 2 ; 1 / q=\theta / 2$, for $0<\theta<1$ (i.e., $1<p<2$ and $q$ is the conjugate exponent to $p$ ), then the Riesz-Thorin interpolation theorem yields

$$
\sum_{\lambda \in \Lambda}\left|\left[f, e_{\lambda}\right]_{L^{p}(\mu)}\right|^{q} \leq \mathcal{C}^{q}\|f\|_{L^{p}(\mu)}^{q} \quad \text { for all } f \in L^{p}(\mu),
$$

where $\mathcal{C} \leq C^{\frac{1}{2} \theta}$. Therefore, $\nu=\sum_{\lambda \in \Lambda} \delta_{\lambda}$ is a $(p, q)$-Bessel measure for $\mu=\frac{1}{|S|} \chi_{S} d x$, and we have $\nu \in \mathcal{B}_{\mathcal{C}^{q}}(\mu)_{(p, q)}$, where $1<p<2$ and $q$ is the conjugate exponent to p. Moreover, $\nu \in \mathcal{B}_{C}(\mu)_{(2,2)}$ and $\nu \in \mathcal{B}_{1}(\mu)_{(1, \infty)}$. On the other hand for every $1<p<2$ and $q$ (the conjugate exponent to $p$ ), $\left\{e_{\lambda}\right\}_{\lambda \in \Lambda}$ is a $q$-Bessel sequence for $L^{p}(\mu)$, with bound $\mathcal{C}^{q}$.

If $S \subset \mathbb{R}^{d}$ is a compact set with positive Lebesgue measure, then by Theorem 3.1, we always have the measure $\mu=\frac{1}{|S|} \chi_{S} d x$ is an F-spectral measure, but regardless of the fact whether it is a spectral measure, it is related to Fuglede's conjecture [11]. In the following example, we will consider a spectral measure of this type.

Example 3.2. Let $\mu=\chi_{\{[0,1] \cup[2,3]\}} d x$. The set of exponential functions $\left\{e_{\lambda}: \lambda \in \Lambda:=\right.$ $\left.\mathbb{Z} \cup \mathbb{Z}+\frac{1}{4}\right\}$ is an orthogonal basis for $L^{2}(\mu)$ (see [9]). We will consider the probability measure $\mu^{\prime}=\frac{1}{2} \chi_{\{[0,1] \cup[2,3]\}} d x$. Then for every $f$ in $L^{2}\left(\mu^{\prime}\right)$, we have $\sum_{\lambda \in \Lambda}\left|\left\langle f, e_{\lambda}\right\rangle_{L^{2}\left(\mu^{\prime}\right)}\right|^{2}=$ $\|f\|_{L^{2}\left(\mu^{\prime}\right)}^{2}$. In addition, for every $f \in L^{1}\left(\mu^{\prime}\right)$, we have $\left\|\left\{\left[f, e_{\lambda}\right]_{L^{1}\left(\mu^{\prime}\right)}\right\}_{\lambda \in \Lambda}\right\|_{\infty} \leq\|f\|_{L^{1}\left(\mu^{\prime}\right)}$. Now by applying the Riesz-Thorin interpolation theorem $\sum_{\lambda \in \Lambda}\left|\left[f, e_{\lambda}\right]_{L^{2}\left(\mu^{\prime}\right)}\right|^{q} \leq\|f\|_{L^{p}\left(\mu^{\prime}\right)}^{q}$, for all $f \in L^{p}\left(\mu^{\prime}\right)$, where $1 \leq p \leq 2$ and $q$ is the conjugate exponent to $p$. Hence, $\nu=\sum_{\lambda \in \Lambda} \delta_{\lambda}$ is a $(p, q)$-Bessel measure for $\mu^{\prime}$, especially $\nu \in \mathcal{B}_{1}\left(\mu^{\prime}\right)_{(p, q)}$, where $1 \leq p \leq 2$ and $q$ is the conjugate exponent to $p$. Besides, $\left\{e_{\lambda}\right\}_{\lambda \in \Lambda}$ is a $q$-Bessel sequence for $L^{p}\left(\mu^{\prime}\right)$ with bound 1 , where $1<p \leq 2$ and $q$ is the conjugate exponent to $p$.

Proposition 3.8. [20] Let $\mu(x)=\phi(x) d x$ be a compactly supported absolutely continuous probability measure. Then $\mu$ is an F-spectral measure if and only if the density function $\phi(x)$ is bounded above and below almost everywhere on the support (see also [8]). 
Corollary 3.4. If the density function of a compactly supported absolutely continuous probability measure $\mu$ is essentially bounded above and below on the support, then the following assertions hold.

(i) There exists an infinite $(p, q)$-Bessel measure $\nu=\sum_{\lambda \in \Lambda_{\mu}} \delta_{\lambda}$ for $\mu$, where $1 \leq p \leq 2$ and $q$ is the conjugate exponent to $p$. Moreover, when $\mu$ is a spectral measure, we have $\nu \in \mathcal{B}_{1}(\mu)_{p, q}$, where $1 \leq p \leq 2$ and $q$ is the conjugate exponent to $p$.

(ii) There exists a q-Bessel sequence $\left\{e_{\lambda}\right\}_{\lambda \in \Lambda_{\mu}}$ for $L^{p}(\mu)$, where $1<p \leq 2$ and $q$ is the conjugate exponent to $p$. In addition, when $\mu$ is a spectral measure, $\left\{e_{\lambda}\right\}_{\lambda \in \Lambda_{\mu}}$ is a $q$-Bessel sequence for $L^{p}(\mu)$ with bound 1 , where $1<p \leq 2$ and $q$ is the conjugate exponent to $p$.

Proof. The conclusion follows from Proposition 3.8 and the Riesz-Thorin interpolation theorem (see the proof of Theorem 3.2 and also, see Example 3.2).

By Proposition 3.3, if $1<p, q<\infty$ and $1 / p+1 / q=1$, then a fixed finite Borel measure $\nu$ is a $(p, q)$-Bessel measure for every finite measure $\mu$, especially $\nu \in$ $\mathcal{B}_{\nu\left(\mathbb{R}^{d}\right)}(\mu)_{(p, q)}$ for all probability measures $\mu$. In the following part, we will give an example of a discrete spectral measure $\mu$ such that it has a finite discrete $(p, q)$ Bessel measure $\nu$ with Bessel bound less than $\nu\left(\mathbb{R}^{d}\right)$, precisely $\nu \in \mathcal{B}_{1}(\mu)_{(p, q)}$, where $1 \leq p \leq 2$ and $q$ is the conjugate exponent to $p$.

Example 3.3. Consider the atomic measure $\mu:=\frac{1}{2}\left(\delta_{0}+\delta_{\frac{1}{2}}\right)$, the set $\left\{e_{l}: l \in L:=\{0,1\}\right\}$ is an orthonormal basis for $L^{2}(\mu)$. Hence $\sum_{l \in L}\left|\left\langle f, e_{l}\right\rangle_{L^{2}(\mu)}\right|^{2}=\|f\|_{L^{2}(\mu)}^{2}$ for all $f \in L^{2}(\mu)$. Moreover, $\left\|\left\{\left[f, e_{l}\right]_{L^{1}(\mu)}\right\}_{l \in L}\right\|_{\infty} \leq\|f\|_{L^{1}(\mu)}$ for every $f$ in $L^{1}(\mu)$. Now by applying the Riesz-Thorin interpolation theorem $\sum_{l \in L}\left|\left[f, e_{l}\right]_{L^{p}(\mu)}\right|^{q} \leq\|f\|_{L^{p}(\mu)}^{q}$, for all $f \in L^{p}(\mu)$, where $1 \leq p \leq 2$ and $q$ is the conjugate exponent to $p$. Therefore, $\left\{e_{l}\right\}_{l \in L}$ is a finite $q$-Bessel sequence for $L^{p}(\mu)$ with bound 1 , and $\nu=\sum_{l \in L} \delta_{l}$ is a finite discrete $(p, q)$ Bessel measure for $\mu$, especially $\nu \in \mathcal{B}_{1}(\mu)_{(p, q)}$, where $1 \leq p \leq 2$ and $q$ is the conjugate exponent to $p$. When $p>2$ and $q$ is the conjugate exponent to $p$, based on Proposition 3.3 $\nu \in \mathcal{B}_{2}(\mu)_{(p, q)}$ and $\left\{e_{l}\right\}_{l \in L}$ is a finite $q$-Bessel sequence for $L^{p}(\mu)$ with bound 2.

Proposition 3.9. [13] Let $\mu=\sum_{c \in C} p_{c} \delta_{c}$ be a discrete probability measure on $\mathbb{R}^{d}$. $\mu$ is an F-spectral measure with an F-spectrum $\Lambda$ if and only if $\# C<\infty$ and $\# \Lambda<\infty$.

Corollary 3.5. Let $1<p, q<\infty$ and $1 / p+1 / q=1$. If $\mu$ is any probability measure, then the following assertions hold.

(i) A finite discrete measure $\nu=\sum_{\lambda \in \Lambda} \delta_{\lambda}$ is a $(p, q)$-Bessel measure for $\mu$, precisely $\nu \in \mathcal{B}_{\nu\left(\mathbb{R}^{d}\right)}(\mu)_{(p, q)}$. If $\mu=\sum_{c \in C} p_{c} \delta_{c}$ and if $\mu$ is an F-spectral measure with the $F$-spectrum $\Lambda$, then for every $1<p \leq 2$ there exists a positive constant $\mathcal{C}$ such that we have $\nu \in \mathcal{B}_{\mathcal{C}}(\mu)_{(p, q)}$ (q is the conjugate exponent to $p$ ). In addition, If $\mu=\sum_{c \in C} p_{c} \delta_{c}$ is a spectral measure with the spectrum $\Lambda$, then we have $\nu \in$ $\mathcal{B}_{1}(\mu)_{(p, q)}$, where $1<p \leq 2$ and $q$ is the conjugate exponent to $p$. 
(ii) A finite sequence $\left\{e_{\lambda}\right\}_{\lambda \in \Lambda}$ is a q-Bessel sequence for $L^{p}(\mu)$ with bound $\nu\left(\mathbb{R}^{d}\right)$ $\left(\nu=\sum_{\lambda \in \Lambda} \delta_{\lambda}\right)$. If $\mu=\sum_{c \in C} p_{c} \delta_{c}$ and if $\mu$ is an F-spectral measure with the $F$ spectrum $\Lambda$, then for every $1<p \leq 2$ there exists a constant $\mathcal{C}$ such that $\left\{e_{\lambda}\right\}_{\lambda \in \Lambda}$ is a $q$-Bessel sequence for $L^{p}(\mu)$ with bound $\mathcal{C}(q$ is the conjugate exponent to $p)$. In addition, If $\mu=\sum_{c \in C} p_{c} \delta_{c}$ is a spectral measure with the spectrum $\Lambda$, then $\left\{e_{\lambda}\right\}_{\lambda \in \Lambda}$ is a $q$-Bessel sequence for $L^{p}(\mu)$ with bound 1 , where $1<p \leq 2$ and $q$ is the conjugate exponent to $p$.

Proof. The conclusion follows from Propositions 3.3, 3.9, 3.2, and the Riesz-Thorin interpolation theorem. In fact, the corollary says that if a probability measure $\mu$ is also a discrete F-spectral measure, then beside the bound $\nu\left(\mathbb{R}^{d}\right)$, we can find other bounds by applying Riesz-Thorin interpolation theorem (where $1<p \leq 2$ and $q$ is the conjugate exponent to $p$ ). As we can see in Example 3.3, for all $\bar{p}>1$ and $q$ (the conjugate exponent to $p$ ), we have $\nu \in \mathcal{B}_{2}(\mu)_{(p, q)}$ and since $\mu$ is a spectral measure with the spectrum $L$, we also have $\nu \in \mathcal{B}_{1}(\mu)_{(p, q)}$, where $1 \leq p \leq 2$ and $q$ is the conjugate exponent to $p$.

Theorem 3.3. [4] Let $R$ be a $d \times d$ expansive integer matrix, $0 \in \mathcal{A} \subset \mathbb{Z}^{d}$. Let $\mu_{\mathcal{A}}$ be an invariant measure associated to the iterated function system

$$
\tau_{a}(x)=R^{-1}(x+a) \quad\left(x \in \mathbb{R}^{d}, a \in \mathcal{A}\right)
$$

and the probabilities $\left(\rho_{a}\right)_{a \in \mathcal{A}}$. Then $\mu$ has an infinite B-spectrum of positive Beurling dimension (Beurling dimension is used as a method of investigating existence of Bessel spectra for singular measures).

Theorem 3.4. Any fractal measure $\mu$ obtained from an affine iterated function system has an infinite discrete $(p, q)$-Bessel measure $\nu$, where $1 \leq p \leq 2$ and $q$ is the conjugate exponent to $p$.

Proof. Suppose that $R$ is a $d \times d$ expansive integer matrix, $0 \in \mathcal{A} \subset \mathbb{Z}^{d}$. If $\mu_{\mathcal{A}}$ is an invariant measure associated to the iterated function system

$$
\tau_{a}(x)=R^{-1}(x+a) \quad\left(x \in \mathbb{R}^{d}, a \in \mathcal{A}\right)
$$

and the probabilities $\left(\rho_{a}\right)_{a \in \mathcal{A}}$, then according to Theorem 3.3 there exists an infinite subset $\Lambda$ of $\mathbb{R}^{d}$ and a constant $B>0$ such that

$$
\sum_{\lambda \in \Lambda}\left|\left\langle f, e_{\lambda}\right\rangle_{L^{2}\left(\mu_{\mathcal{A}}\right)}\right|^{2} \leq B\|f\|_{L^{2}\left(\mu_{\mathcal{A}}\right)}^{2} \quad \text { for all } f \in L^{2}\left(\mu_{\mathcal{A}}\right)
$$

We also have $\left\|\left\{\left[f, e_{\lambda}\right]_{L^{1}\left(\mu_{\mathcal{A}}\right)}\right\}_{\lambda \in \Lambda}\right\|_{\infty} \leq\|f\|_{L^{1}\left(\mu_{\mathcal{A}}\right)}$, for every $f \in L^{1}\left(\mu_{\mathcal{A}}\right)$. Now if $1 / p=1-\theta / 2 ; 1 / q=\theta / 2$, for $0<\theta<1$ (i.e., $1<p<2$ and $q$ is the conjugate exponent to $p$ ), then the Riesz-Thorin interpolation theorem yields

$$
\sum_{\lambda \in \Lambda}\left|\left[f, e_{\lambda}\right]_{L^{p}\left(\mu_{\mathcal{A}}\right)}\right|^{q} \leq B^{\prime q}\|f\|_{L^{p}\left(\mu_{\mathcal{A}}\right)}^{q} \quad \text { for all } f \in L^{p}\left(\mu_{\mathcal{A}}\right)
$$


where $B^{\prime} \leq B^{\frac{1}{2} \theta}$. Thus, $\nu=\sum_{\lambda \in \Lambda} \delta_{\lambda}$ is a $(p, q)$-Bessel measure for $\mu_{\mathcal{A}}$, and $\nu \in \mathcal{B}_{B^{\prime q}}\left(\mu_{\mathcal{A}}\right)_{(p, q)}$, where $1<p<2$ and $q$ is the conjugate exponent to $p$. Moreover, we have $\nu \in \mathcal{B}_{B}\left(\mu_{\mathcal{A}}\right)_{(2,2)}$ and $\nu \in \mathcal{B}_{1}\left(\mu_{\mathcal{A}}\right)_{(1, \infty)}$. On the other hand, for every $1<p<2$ and $q$ (the conjugate exponent to $p$ ), $\left\{e_{\lambda}\right\}_{\lambda \in \Lambda}$ is a $q$-Bessel sequence for $L^{p}\left(\mu_{\mathcal{A}}\right)$ with bound $B^{\prime q}$.

If a measure $\mu$ is an F-spectral measure, then it must be of pure type, i.e., $\mu$ is either discrete, singular continuous or absolutely continuous $[19,13]$. The case when the measure $\mu$ is singular continuous, is not precisely known. The first known example of a singular continuous spectral measure supported on a non-integer dimension set (a fractal measure), was given by Jorgensen and Pedersen [16]. They showed that the measure $\mu_{4}$ (the Cantor measures supported on Cantor set of $1 / 4$ contraction), is spectral. A spectrum of $\mu_{4}$ is $\Lambda=\left\{\sum_{m=0}^{k} 4^{m} d_{m}: d_{m} \in\{0,1\}, k \in \mathbb{N}\right\}$. They also showed that $\mu_{2 k}$ (the Cantor measures with even contraction ratio) is spectral, but $\mu_{2 k+1}$ (the Cantor measures with odd contraction ratio) is not.

Remark 3.3. Since Cantor type measures are fractal measures, by applying Theorem 3.4 one can obtain that every Cantor type measure $\mu$ admits a $(p, q)$-Bessel measure $\nu=$ $\sum_{\lambda \in \Lambda_{\mu}} \delta_{\lambda}$, where $1 \leq p \leq 2$ and $q$ is the conjugate exponent to $p$. Moreover, for every spectral Cantor type measure $\mu_{2 k}$, we have $\nu \in \mathcal{B}_{1}\left(\mu_{2 k}\right)_{p, q}$, where $1 \leq p \leq 2$ and $q$ is the conjugate exponent to $p$.

In [21] the author presents a method for constructing many examples of continuous measures $\mu$ (including fractal ones) which have components of different dimensions, but nevertheless they are F-spectral measures. In the following part, we will provide some results by [21]. By applying the Riesz-Thorin interpolation theorem, one can obtain infinite discrete $(p, q)$-Bessel measures $\nu=\sum_{\lambda \in \Lambda_{\mu}} \delta_{\lambda}$ (where $1 \leq p \leq 2$ and $q$ is the conjugate exponent to $p$ ), for such F-spectral measures $\mu$.

Definition 3.1. [[21]] Let $\mu$ and $\mu^{\prime}$ be positive and finite measures on $\mathbb{R}^{n}$ and $\mathbb{R}^{m}$, respectively. A mixed type measure $\rho$ is a measure which is constructed on $\mathbb{R}^{n+m}=\mathbb{R}^{n} \times \mathbb{R}^{m}$ and defined by

$$
\rho=\mu \times \delta_{0}+\delta_{0} \times \mu^{\prime},
$$

where $\delta_{0}$ denotes the Dirac measure at the origin. Equivalently, the measure $\rho$ may be defined by the requirement that

$$
\int_{\mathbb{R}^{n} \times \mathbb{R}^{m}} f(x, y) d \rho(x, y)=\int_{\mathbb{R}^{n}} f(x, 0) d \mu(x)+\int_{\mathbb{R}^{m}} f(0, y) d \mu^{\prime}(y),
$$

for every continuous, compactly supported function $f$ on $\mathbb{R}^{n} \times \mathbb{R}^{m}$.

Theorem 3.5. [21] Let $\mu$ and $\mu^{\prime}$ be continuous F-spectral measures. Then the mixed type measure $\rho=\mu \times \delta_{0}+\delta_{0} \times \mu^{\prime}$ is also an F-spectral measure. 
Theorem 3.6. [21] If $\mu$ is the sum of the $k$-dimensional area measure on $[0,1]^{k} \times$ $\{0\}^{d-k}$, and the $j$-dimensional area measure on $\{0\}^{d-j} \times[0,1]^{j}$ where $1 \leq j, k \leq$ $d-1$, then $\mu$ is an $F$-spectral measure.

The following theorem provides many examples of single dimensional measures which are F-spectral measures:

Theorem 3.7. [21] Let $\phi: \mathbb{R}^{k} \rightarrow \mathbb{R}^{d-k}$ be a smooth function $(1 \leq k \leq d-1)$. If $\mu$ is the $k$-dimensional area measure on a compact subset of the graph $\{(x, \phi(x)): x \in$ $\left.\mathbb{R}^{k}\right\}$ of $\phi$, then $\mu$ is an F-spectral measure.

The next proposition shows that if $1<p, q<\infty$ and $1 / p+1 / q=1$, then considering any countable subset (finite or infinite) $\Lambda$ of $\mathbb{R}^{d}$, one can obtain tight $(p, q)$-frame measures and $(p, q)$-Plancherel measures $\nu_{\Lambda}$ for $\delta_{0}$. In addition, there exists tight and Parseval $q$-frames for $L^{p}\left(\delta_{0}\right)$.

Proposition 3.10. Suppose that $1<p, q<\infty$ and $1 / p+1 / q=1$. Then there exists a measure $\mu$ which admits tight $(p, q)$-frame measures and $(p, q)$-Plancherel measures. Moreover, there exists tight and Parseval q-frames for $L^{p}(\mu)$.

Proof. Let $\mu=\delta_{0}$. For a countable subset $\Lambda$ of $\mathbb{R}^{d}$, Let $\nu_{\Lambda}=\sum_{\lambda \in \Lambda} c_{\lambda} \delta_{\lambda}$ where $c_{\lambda}>0$.

If $\sum_{\lambda \in \Lambda} c_{\lambda}=m \neq 1$, then for all $f \in L^{p}(\mu)$,

$$
\int_{\mathbb{R}^{d}}\left|\left[f, e_{t}\right]_{L^{p}(\mu)}\right|^{q} d \nu(t)=\sum_{\lambda \in \Lambda} c_{\lambda}|f(0)|^{q}=m\|f\|_{L^{p}(\mu)}^{q} .
$$

If $\sum_{\lambda \in \Lambda} c_{\lambda}=1$, then for all $f \in L^{p}(\mu)$,

$$
\int_{\mathbb{R}^{d}}\left|\left[f, e_{t}\right]_{L^{p}(\mu)}\right|^{q} d \nu(t)=\sum_{\lambda \in \Lambda} c_{\lambda}|f(0)|^{q}=\|f\|_{L^{p}(\mu)}^{q}
$$

On the other hand, for all $f \in L^{p}(\mu)$ we have

$$
\int_{\mathbb{R}^{d}}\left|\left[f, e_{t}\right]_{L^{p}(\mu)}\right|^{q} d \nu(t)=\sum_{\lambda \in \Lambda} c_{\lambda}\left|\left[f, e_{\lambda}\right]_{L^{p}(\mu)}\right|^{q}=\sum_{\lambda \in \Lambda}\left|\left[f, \sqrt[q]{c_{\lambda}} e_{\lambda}\right]_{L^{p}(\mu)}\right|^{q} .
$$

Hence, If $\sum_{\lambda \in \Lambda} c_{\lambda}=m \neq 1$, then $\left\{\sqrt[q]{c_{\lambda}} e_{\lambda}\right\}_{\lambda \in \Lambda}$ is a tight $q$-frame for $L^{p}(\mu)$, and If $0<c_{\lambda}<1, \sum_{\lambda \in \Lambda} c_{\lambda}=1$, then $\left\{\sqrt[q]{c_{\lambda}} e_{\lambda}\right\}_{\lambda \in \Lambda}$ is a Parseval $q$-frame for $L^{p}(\mu)$.

Proposition 3.11. Let $\mu$ be a finite Borel measure and let $B$ be a positive constant. Then there exists a $(p, q)$-Bessel measure $\nu$ for $\mu$ for all $1<p, q<\infty$ and $1 / p+1 / q=1$, such that $\nu \in \mathcal{B}_{B}(\mu)_{p, q}$. In addition, for every $1<p, q<\infty$ and $1 / p+1 / q=1$, there exists a $q$-Bessel sequence with bound $B$ for $L^{p}(\mu)$. 
Proof. Let $\nu=\sum_{i \in I} c_{i} \delta_{\lambda_{i}}$ for some $\lambda_{i} \in \mathbb{R}^{d}$ such that $\sum_{i \in I} c_{i} \leq \frac{B}{\mu\left(\mathbb{R}^{d}\right)}$. Let $p>1$ and $f \in L^{p}(\mu)$. If $q$ is the conjugate exponent to $p$, then by applying Holder's inequality we have

$$
\int_{\mathbb{R}^{d}}\left|\left[f, e_{t}\right]_{L^{p}(\mu)}\right|^{q} d \nu(t) \leq \sum_{i \in I} c_{i}\|f\|_{L^{p}(\mu)}^{q} \mu\left(\mathbb{R}^{d}\right) \leq B\|f\|_{L^{p}(\mu)}^{q} .
$$

Hence $\nu \in \mathcal{B}_{B}(\mu)_{p, q}$.

Since

$$
\sum_{i \in I}\left|\left[f, \sqrt[q]{c_{i}} e_{\lambda_{i}}\right]_{L^{p}(\mu)}\right|^{q}=\sum_{i \in I} c_{i}\left|\left[f, e_{\lambda_{i}}\right]_{L^{p}(\mu)}\right|^{q}=\int_{\mathbb{R}^{d}}\left|\left[f, e_{t}\right]_{L^{p}(\mu)}\right|^{q} d \nu(t),
$$

the second statement follows from (3.1).

All infinite $(p, q)$-Bessel measures $\nu$ we observed were discrete. Now the question is whether we can find a finite measure $\mu$ which admits a continuous infinite $(p, q)$ Bessel measure $\nu$. In the following we show that the answer is affirmative (see also Example 4.1).

Proposition 3.12. If $\nu=\lambda$ (the Lebesgue measure on $\mathbb{R}^{d}$ ) and $\mu=\left.\lambda\right|_{[0,1]^{d}}$, then $\lambda$ is $a(p, q)$-Bessel measure for $\mu$ where $1 \leq p \leq 2$ and $q$ is the conjugate exponent to $p$.

Proof. According to Plancherel's theorem the following equation is satisfied:

$$
\int_{\mathbb{R}^{d}}|\hat{f}(t)|^{2} d \lambda(t)=\int_{\mathbb{R}^{d}}|f(x)|^{2} d \lambda(x) \quad \text { for all } f \in L^{2}(\lambda) .
$$

If $f$ is supported on $[0,1]^{d}$, then

$$
\int_{\mathbb{R}^{d}}|\widehat{f d \mu}|^{2} d \lambda(t)=\int_{\mathbb{R}^{d}}|f(x)|^{2} d \mu(x) \quad \text { for all } f \in L^{2}(\mu) .
$$

Moreover, we have $\|\widehat{f d \mu}\|_{\infty} \leq\|f\|_{L^{1}(\mu)}$ for all $f$ in $L^{1}(\mu)$. Now by applying the Riesz-Thorin interpolation theorem

$$
\int_{\mathbb{R}^{d}}|\widehat{f d \mu}|^{q} d \lambda(t) \leq\|f\|_{L^{p}(\mu)}^{q} \quad \text { for all } f \in L^{p}(\mu),
$$

where $1 \leq p \leq 2$ and $q$ is the conjugate exponent to $p$. Hence $\lambda \in \mathcal{B}_{1}(\mu)_{p, q}$.

(likewise, for every $\mu=\left.\lambda\right|_{S}$, where $S$ is a subset of $\mathbb{R}^{d}$ with finite Lebesgue measure we have $\lambda \in \mathcal{B}_{1}(\mu)_{p, q}$, where $1 \leq p \leq 2$ and $q$ is the conjugate exponent to p)

Corollary 3.6. The measure $\mu=\left.\lambda\right|_{[0,1]^{d}}$ has infinite continuous and discrete $(p, q)$-Bessel measures, where $1 \leq p \leq 2$ and $q$ is the conjugate exponent to $p$. More precisely, if $\nu_{1}=\sum_{t \in \mathbb{Z}^{d}} \delta_{t}$ and $\nu_{2}=\lambda$, then $\nu_{1}, \nu_{2} \in \mathcal{B}_{1}(\mu)_{p, q}$. 
Proof. The conclusion follows from Example 3.1 and Proposition 3.12.

Corollary 3.7. Every $\mu=\left.\lambda\right|_{S}$, where $S$ is a subset of $\mathbb{R}^{d}$ with finite Lebesgue measure, has infinite continuous and discrete $(p, q)$-Bessel measures, where $1 \leq p \leq$ 2 and $q$ is the conjugate exponent to $p$.

Proof. The approach is similar to Proposition 3.12 and Theorem 3.2.

\section{Properties and Structural Results}

In this section our assertions are based on the results by Dutkay, Han, and Weber from [5]. We generalize the results and we give some of the proofs for completeness.

Proposition 4.1. Let $\mu$ be a Borel probability measure. Let $1<p, q<\infty$ and $1 / p+1 / q=1$. If $\nu$ is a $(p, q)$-Bessel measure for $\mu$, then there exists a constant $C$ such that $\nu(K) \leq C \operatorname{diam}(K)^{d}$ for any compact subset $K$ of $\mathbb{R}^{d}$. Accordingly, $\nu$ is $\sigma$-finite.

Proof. It is easy to check that $\widehat{d \mu}: \mathbb{R}^{d} \rightarrow \mathbb{C}$ is uniformly continuous and $\widehat{d \mu}(0)=$ $\mu\left(\mathbb{R}^{d}\right)=1$. So for every $\eta>0$ there exists $\epsilon>0$ such that for $x \in \mathrm{B}(0, \epsilon)$ we have $|\widehat{d \mu}(0)|-|\widehat{d \mu}(x)| \leq|\widehat{d \mu}(0)-\widehat{d \mu}(x)| \leq \eta$, and then $|\widehat{d \mu}(x)| \geq 1-\eta$. If $\delta:=(1-\eta)^{q}$, then $|\widehat{d \mu}(x)|^{q} \geq \delta$ for $x \in \mathrm{B}(0, \epsilon)$. Thus, for any $t \in \mathbb{R}^{d}$,

$$
\begin{aligned}
B=B\left\|e_{t}\right\|_{L^{p}(\mu)}^{q} & \geq \int_{\mathbb{R}^{d}}\left|\left[e_{t}, e_{x}\right]\right|^{q} d \nu(x)=\int_{\mathbb{R}^{d}}\left|\left[1, e_{x-t}\right]\right|^{q} d \nu(x) \\
& =\int_{\mathbb{R}^{d}}|\widehat{d \mu}(x-t)|^{q} d \nu(x) \geq \int_{\mathrm{B}(t, \epsilon)}|\widehat{d \mu}(x-t)|^{q} d \nu(x) \\
& \geq \nu(\mathrm{B}(t, \epsilon)) \delta .
\end{aligned}
$$

Now Let $K \subseteq \mathbb{R}^{d}$ be compact and $r=\operatorname{diam}(K)$. Then there exists a point $x=$ $\left(x_{1}, \ldots, x_{d}\right)$ in $\mathbb{R}^{d}$ such that $K \subset \prod_{i=1}^{d}\left[x_{i}-r, x_{i}+r\right]$. We may assume that $\epsilon<2 r$ and $2 r / \epsilon \in \mathbb{N}$. Let $M=2 r / \epsilon$. We have $\prod_{i=1}^{d}\left[x_{i}-r, x_{i}+r\right]=\bigcup_{\alpha=1}^{M^{d}} C_{\alpha}$ where $C_{\alpha} \mathrm{s}$ are d-dimensional cubes of side length $\epsilon$. For any $\alpha \in\left\{1, \ldots, M^{d}\right\}$, let $t_{\alpha}$ be the center point of $C_{\alpha}$. Then $C_{\alpha} \subset \mathrm{B}\left(t_{\alpha}, \epsilon\right)$. Now if $C:=(2 / \epsilon)^{d} B / \delta$, then

$$
\nu(K) \leq \nu\left(\bigcup_{\alpha=1}^{M^{d}} \mathrm{~B}\left(t_{\alpha}, \epsilon\right)\right) \leq \sum_{\alpha=1}^{M^{d}} \nu\left(\mathrm{B}\left(t_{\alpha}, \epsilon\right)\right) \leq\left(\frac{2 r}{\epsilon}\right)^{d} \frac{B}{\delta}=r^{d}\left(\frac{2}{\epsilon}\right)^{d} \frac{B}{\delta}=C r^{d} .
$$

Hence the assertion follows.

Theorem 4.1. Let $1<p, q<\infty$ and $1 / p+1 / q=1$. Let $B>A>0$. Then the set $\mathcal{F}_{A, B}(\mu)_{p, q}$ is empty for some finite compactly supported Borel measures $\mu$. 
Proof. Let $\mu=\chi_{[0,1]} d x+\delta_{2}$. Suppose $\nu \in \mathcal{F}_{A, B}(\mu)_{p, q}$. Let $f:=\chi_{\{2\}}$. Then $\|f\|_{L^{p}(\mu)}=1$ and $\left|\left[f, e_{t}\right]_{L^{p}(\mu)}\right|=1$ for all $t \in \mathbb{R}$. In addition, the upper bound implies that $\nu(\mathbb{R}) \leq B<\infty$. Then from the inner regularity of Borel measures we obtain that for any $\epsilon>0$ there exists a compact set $K \subset \mathbb{R}$ and a positive constant $R$ such that $\nu(\mathbb{R})-\epsilon<K \leq \nu(\mathrm{B}(0, R))$. Therefore, $\nu(\mathbb{R} \backslash \mathrm{B}(0, R))<\epsilon$.

Choose some $T$ large, arbitrary and let $g(x):=e^{-2 \pi i T x} \chi_{[0,1]}$. Then

$$
\left|\left[g, e_{t}\right]_{L^{p}(\mu)}\right|^{q}=\left|\int_{[0,1]} e^{-2 \pi i(T+t) x} d x\right|^{q}=\left|\frac{\sin (\pi(T+t))}{\pi(T+t)}\right|^{q} \quad(t \in \mathbb{R}) .
$$

The substitution $z:=-2 \pi x$ gives the last equality. Consequently, for all $t \in \mathbb{R}$, $\left|\left[g, e_{t}\right]_{L^{p}(\mu)}\right|^{q} \leq 1$ and if we take $T \geq 2 R$, then for all $t \in(-R, R)$ we have

$$
\left|\left[g, e_{t}\right]_{L^{p}(\mu)}\right|^{q} \leq \frac{1}{\pi^{q}(T-R)^{q}} .
$$

Hence from the lower bound we obtain

$$
\begin{aligned}
A=A\|g\|_{L^{p}(\mu)}^{q} & \leq \int_{\mathbb{R}}\left|\left[g, e_{t}\right]_{L^{p}(\mu)}\right|^{q} d \nu(t) \\
& =\int_{\mathrm{B}(0, R)}\left|\left[g, e_{t}\right]_{L^{p}(\mu)}\right|^{q} d \nu(t)+\int_{\mathbb{R} \backslash \mathrm{B}(0, R)}\left|\left[g, e_{t}\right]_{L^{p}(\mu)}\right|^{q} d \nu(t) \\
& \leq \frac{1}{\pi^{q}(T-R)^{q}} \cdot \nu(\mathbb{R})+\epsilon .
\end{aligned}
$$

Now if $T \rightarrow \infty$ and $\epsilon \rightarrow 0$, then $A=0$. This is a contradiction.

The next proposition shows that if there exists a $(p, q)$-Bessel/frame measure, then many others can be constructed.

Proposition 4.2. Let $\mu$ be a finite Borel measure and $A, B$ be positive constants. Let $1<p, q<\infty$ and $1 / p+1 / q=1$. Then both sets $\mathcal{B}_{B}(\mu)_{p, q}$ and $\mathcal{F}_{A, B}(\mu)_{p, q}$ are convex and closed under convolution with Borel probability measures.

Proof. Let $\nu_{1}, \nu_{2} \in \mathcal{B}_{B}(\mu)_{p, q}$ and $0<\lambda<1$. For all $f \in L^{p}(\mu)$,

$$
\begin{aligned}
\int_{\mathbb{R}^{d}}|\widehat{f d \mu}|^{q} d\left(\lambda \nu_{1}+(1-\lambda) \nu_{2}\right) & =\lambda \int_{\mathbb{R}^{d}}|\widehat{f d \mu}|^{q} d \nu_{1}+(1-\lambda) \int_{\mathbb{R}^{d}}|\widehat{f d \mu}|^{q} d \nu_{2} \\
& \leq B\|f\|_{L^{p}(\mu)}^{q} .
\end{aligned}
$$

Then $\lambda \nu_{1}+(1-\lambda) \nu_{2} \in \mathcal{B}_{B}(\mu)_{p, q}$. Similarly, if $\nu_{1}, \nu_{2} \in \mathcal{F}_{A, B}(\mu)_{p, q}$, then we have $\lambda \nu_{1}+(1-\lambda) \nu_{2} \in \mathcal{F}_{A, B}(\mu)_{p, q}$.

Let $s \in \mathbb{R}^{d}$. Then for all $f \in L^{p}(\mu)$,

$$
\left\|e_{s} f\right\|_{L^{p}(\mu)}^{p}=\int_{\mathbb{R}^{d}}\left|e_{s}(x) f(x)\right|^{p} d \mu(x)=\int_{\mathbb{R}^{d}}|f(x)|^{p} d \mu(x)=\|f\|_{L^{p}(\mu)}^{p} .
$$


In addition, let $\nu \in \mathcal{B}_{B}(\mu)_{p, q}$ and let $\rho$ be a Borel probability measure on $\mathbb{R}^{d}$. Then for any $t \in \mathbb{R}^{d}$ and $f \in L^{p}(\mu)$,

$$
\begin{aligned}
{\left[e_{-s} f, e_{t}\right]_{L^{p}(\mu)} } & =\int_{\mathbb{R}^{d}} e_{-s}(x) f(x) e^{-2 \pi i t \cdot x} d \mu(x)=\int_{\mathbb{R}^{d}} f(x) e^{-2 \pi i(s+t) \cdot x} d \mu(x) \\
& =\left[f, e_{s+t}\right]_{L^{p}(\mu)} .
\end{aligned}
$$

Therefore,

$$
\begin{aligned}
\int_{\mathbb{R}^{d}}\left|\left[f, e_{t}\right]_{L^{p}(\mu)}\right|^{q} d \nu * \rho(t) & =\int_{\mathbb{R}^{d}} \int_{\mathbb{R}^{d}}\left|\left[f, e_{t+s}\right]_{L^{p}(\mu)}\right|^{q} d \nu(t) d \rho(s) \\
& =\int_{\mathbb{R}^{d}} \int_{\mathbb{R}^{d}}\left|\left[e_{-s} f, e_{t}\right]_{L^{p}(\mu)}\right|^{q} d \nu(t) d \rho(s) \\
& \leq \int_{\mathbb{R}^{d}} B\left\|e_{-s} f\right\|_{L^{p}(\mu)}^{q} d \rho(s)=B \int_{\mathbb{R}^{d}}\|f\|_{L^{p}(\mu)}^{q} d \rho(s) \\
& =B\|f\|_{L^{p}(\mu)}^{q} .
\end{aligned}
$$

For $\nu \in \mathcal{F}_{A, B}(\mu)_{p, q}$ one can obtain the lower bound analogously.

Corollary 4.1. Let $1<p, q<\infty$ and $1 / p+1 / q=1$. If there exists a $(p, q)$ Bessel/frame measure for $\mu$, then there exists one which is absolutely continuous with respect to the Lebesgue measure and whose Radon-Nikodym derivative is $C^{\infty}$.

Proof. Let $\nu$ be a $(p, q)$-Bessel/frame measure for $\mu$. Convoluting $\nu$ with the Lebesgue measure on $[0,1]$ we have

$$
\begin{aligned}
\nu * \chi_{[0,1]} d \lambda(E) & =\int_{\mathbb{R}} \int_{\mathbb{R}} \chi_{E}(x+y) d \nu(x) \chi_{[0,1]}(y) d \lambda(y) \\
& =\int_{\mathbb{R}} \int_{\mathbb{R}} \chi_{E}(t) \chi_{[0,1]}(t-x) d \nu(x) d \lambda(t-x) \\
& =\int_{\mathbb{R}} \chi_{E}(t) \nu([t-1, t]) d \lambda(t)=\int_{E} \nu([t-1, t]) d \lambda(t),
\end{aligned}
$$

where $E$ is any Borel subset of $\mathbb{R}$. Thus, we obtained a $(p, q)$-Bessel/frame measure for $\mu$ which is absolutely continuous with respect to the Lebesgue measure.

Now consider the following two propositions from [10].

(i) If $d \nu=f d \lambda$ and $d \mu=g d \lambda$, then $d(\nu * \mu)=(f * g) d \lambda$.

(ii) If $f \in L^{1}$ (or $f$ is locally integrable on $\mathbb{R}^{d}$ ), $g \in C^{k}$, and $\partial^{\alpha} g$ is bounded for $|\alpha| \leq k$, then $f * g \in C^{k}$ and $\partial^{\alpha}(f * g)=f *\left(\partial^{\alpha} g\right)$ for $|\alpha| \leq k$.

Let $g \geq 0$ be a compactly supported $C^{\infty}$-function with $\int g(t) d \lambda(t)=1$. Let $d \nu_{0}=\nu * \chi_{[0,1]} d \lambda$ and $d \mu_{0}=g d \lambda$. Then we have $d\left(\nu_{0} * \mu_{0}\right)=(\nu([\cdot-1, \cdot]) * g) d \lambda$ and $\nu([\cdot-1, \cdot]) * g \in C^{\infty}$.

Definition 4.1. [[5]] A sequence of Borel probability measures $\left\{\lambda_{n}\right\}$ is called an approximate identity if

$$
\sup \left\{\|t\|: t \in \operatorname{supp} \lambda_{n}\right\} \rightarrow 0 \quad \text { as } \quad n \rightarrow \infty .
$$


Lemma 4.1. [5] Let $\left\{\lambda_{n}\right\}$ be an approximate identity. If $f$ is a continuous function on $\mathbb{R}^{d}$, then for any $x \in \mathbb{R}^{d}$, we have $\int f(x+t) d \lambda_{n}(t) \rightarrow f(x)$ as $n \rightarrow \infty$.

By Proposition 4.2, if $\nu$ is a $(p, q)$-Bessel/frame measure for $\mu$, then $\nu * \rho$ is also a $(p, q)$-Bessel/frame measure for $\mu$ with the same bound(s), where $\rho$ is any Borel probability measure. An obvious question is under what conditions the converse is true. The next theorem gives an answer.

Theorem 4.2. Let $1<p, q<\infty$ and $1 / p+1 / q=1$. Let $\left\{\lambda_{n}\right\}$ be an approximate identity. Suppose $\nu$ is a $\sigma$-finite Borel measure, and suppose all measures $\nu * \lambda_{n}$ are $(p, q)$-Bessel/frame measures for $\mu$ with uniform bounds, independent of $n$. Then $\nu$ is a $(p, q)$-Bessel/frame measure for $\mu$.

Proof. Take $f \in L^{p}(\mu)$. Since $\left|[f, e .]_{L^{p}(\mu)}\right|^{q}$ (or $|\widehat{f d \mu}|^{q}$ ) is continuous on $\mathbb{R}^{d}$, by Lemma 4.1 and Fatou's lemma we have

$$
\begin{aligned}
\int_{\mathbb{R}^{d}}\left|\left[f, e_{x}\right]_{L^{p}(\mu)}\right|^{q} d \nu(x) & \leq \liminf _{n} \int_{\mathbb{R}^{d}} \int_{\mathbb{R}^{d}}\left|\left[f, e_{x+t}\right]_{L^{p}(\mu)}\right|^{q} d \lambda_{n}(t) d \nu(x) \\
& =\liminf _{n} \int_{\mathbb{R}^{d}}\left|\left[f, e_{y}\right]_{L^{p}(\mu)}\right|^{q} d\left(\nu * \lambda_{n}\right)(y) \\
& \leq B\|f\|_{L^{p}(\mu)}^{q} .
\end{aligned}
$$

Hence $\nu$ is a $(p, q)$-Bessel measure with the same bound $B$ as $\nu * \lambda_{n}$.

Now showing that

$$
\int_{\mathbb{R}^{d}}\left|\left[f, e_{x}\right]_{L^{p}(\mu)}\right|^{q} d\left(\nu * \lambda_{n}\right) \rightarrow \int_{\mathbb{R}^{d}}\left|\left[f, e_{x}\right]_{L^{p}(\mu)}\right|^{q} d \nu
$$

gives the lower bound (see [5]).

We need the following two propositions from [5] to present a general way of constructing $(p, q)$-Bessel/frame measures for a given measure.

Proposition 4.3. [5] Let $\mu$ and $\mu^{\prime}$ be Borel probability measures. For $f \in L^{1}(\mu)$, the measure $(f d \mu) * \mu^{\prime}$ is absolutely continuous w.r.t. $\mu * \mu^{\prime}$ and if the RadonNikodym derivative is denoted by $P f$, then

$$
P f=\frac{(f d \mu) * \mu^{\prime}}{d\left(\mu * \mu^{\prime}\right)} .
$$

Proposition 4.4. [5] Let $\mu, \mu^{\prime}$ be two Borel probability measures and $1 \leq p \leq \infty$. if $f \in L^{p}(\mu)$, then the function $P f$ is in $L^{p}\left(\mu * \mu^{\prime}\right)$ and

$$
\|P f\|_{L^{p}\left(\mu * \mu^{\prime}\right)} \leq\|f\|_{L^{p}(\mu)} .
$$

Now we will show that if a convolution of two measures admits a $(p, q)$-Bessel/frame measure, then one can obtain a $(p, q)$-Bessel/frame measure for one of the measures in the convolution by using the Fourier transform of the other measure in the convolution. 
Proposition 4.5. Let $\mu, \mu^{\prime}$ be two Borel probability measures. Let $1<p, q<\infty$ and $1 / p+1 / q=1$. If $\nu$ is a $(p, q)$-Bessel measure for $\mu * \mu^{\prime}$, then $\left|\hat{\mu}^{\prime}\right|^{q} d \nu$ is a $(p, q)$-Bessel measure for $\mu$ with the same bound.

If in addition $\nu$ is a $(p, q)$-frame measure for $\left(\mu * \mu^{\prime}\right)$ with bounds $A$ and $B$, and for all $f \in L^{p}(\mu), c\|f\|_{L^{p}(\mu)}^{q} \leq\|P f\|_{L^{p}\left(\mu * \mu^{\prime}\right)}^{q}$, then $\left|\hat{\mu}^{\prime}\right|^{q} d \nu$ is a $(p, q)$-frame measure for $\mu$ with bounds $c A$ and $B$.

Proof. If $\mu, \nu \in M\left(\mathbb{R}^{d}\right)$, then $\widehat{\mu * \nu}=\hat{\mu} \cdot \hat{\nu}$ (see[10]). Take $f \in L^{p}(\mu)$. Then

$$
\int_{\mathbb{R}^{d}}|\widehat{(f d \mu)}|^{q} \cdot\left|\hat{\mu}^{\prime}\right|^{q} d \nu=\int_{\mathbb{R}^{d}} \mid\left(\left.f \widehat{d \mu) *} \mu^{\prime}\right|^{q} d \nu=\int_{\mathbb{R}^{d}}\left|P f \widehat{d\left(\mu * \mu^{\prime}\right)}\right|^{q} d \nu .\right.
$$

Thus, we have

$$
\begin{aligned}
c A\|f\|_{L^{p}(\mu)}^{q} & \leq A\|P f\|_{L^{p}\left(\mu * \mu^{\prime}\right)}^{q} \leq \int_{\mathbb{R}^{d}}\left|\operatorname{Pf} \widehat{d\left(\mu * \mu^{\prime}\right)}\right|^{q} d \nu \\
& \leq B\|P f\|_{L^{p}\left(\mu * \mu^{\prime}\right)}^{q} \leq B\|f\|_{L^{p}(\mu)}^{q} .
\end{aligned}
$$

Now by Proposition 4.5, we will show that there exists a singular continuous measure which admits continuous and discrete $(p, q)$-Bessel measures.

Example 4.1. Let $\lambda$ be the Lebesgue measure on $\mathbb{R}$ and $\mu=\left.\lambda\right|_{[0,1]}$. If $\mu_{4}$ is the invariant measure for the affine IFS with $R=4$ and $\mathcal{A}=\{0,2\}$, and if $\mu_{4}^{\prime}$ is the invariant measure for the affine IFS with $R=4$ and $\mathcal{A}^{\prime}=\{0,1\}$, then convolution of measures $\mu_{4}$ and $\mu_{4}^{\prime}$ is the Lebesgue measure on $[0,1]$ (see Corollary 4.7 from [5]). By Corollary 3.6, $\nu_{1}=\sum_{t \in \mathbb{Z}} \delta_{t}$ and $\nu_{2}=\lambda$ are in $\mathcal{B}_{1}(\mu)_{p, q}$, where $1 \leq p \leq 2$ and $q$ is the conjugate exponent to $p$. Hence by Proposition $4.5, \nu_{1}^{\prime}=\sum_{t \in \mathbb{Z}}\left|\widehat{\mu_{4}^{\prime}}(t)\right|^{2} \delta_{t}$ and $\nu_{2}^{\prime}=\left|\widehat{\mu_{4}^{\prime}}(x)\right|^{2} d \lambda(x)$ are in $\mathcal{B}_{1}\left(\mu_{4}\right)_{p, q}$, where $1 \leq p \leq 2$ and $q$ is the conjugate exponent to $p$.

In the next theorem, we have some stability results. In fact, this theorem is a generalization of Proposition 3.5.

Theorem 4.3. Let $\mu$ be a compactly supported Borel probability measure. Let $1<p, q<\infty$ and $1 / p+1 / q=1$. If $\nu$ is a $(p, q)$-Bessel measure for $\mu$, then for any $r>0$ there exists a constant $D>0$ such that

$$
\int_{\mathbb{R}^{d}} \sup _{|y| \leq r}\left|\left[f, e_{x+y}\right]_{L^{p}(\mu)}\right|^{q} d \nu(x) \leq D\|f\|_{L^{p}(\mu)}^{q}, \quad \text { for all } f \in L^{p}(\mu) .
$$

If $\nu$ is a $(p, q)$-frame measure for $\mu$, then there exist constants $\delta>0$ and $C>0$ such that

$$
C\|f\|_{L^{p}(\mu)}^{q} \leq \int_{\mathbb{R}^{d}} \inf _{|y| \leq \delta}\left|\left[f, e_{x+y}\right]_{L^{p}(\mu)}\right|^{q} d \nu(x), \quad \text { for all } f \in L^{p}(\mu) .
$$

Proof. The approach is completely similar to the proof of Theorem 2.10 from [5]. 
We show that by using this stability of $(p, q)$-frame measures, one can obtain atomic $(p, q)$-frame measures from a general $(p, q)$-frame measure.

Definition 4.2. Let $Q=[0,1)^{d}$ and $r>0$. If $\nu$ is a Borel measure on $\mathbb{R}^{d}$ and if $\left(x_{k}\right)_{k \in \mathbb{Z}^{d}}$ is a set of points such that for all $k \in \mathbb{Z}^{d}$ we have $x_{k} \in r(k+Q)$ and $\nu(r(k+Q))<\infty$, then a discretization of the measure $\nu$ is defined by

$$
\nu^{\prime}:=\sum_{k \in \mathbb{Z}^{d}} \nu(r(k+Q)) \delta_{x_{k}} .
$$

Theorem 4.4. Let $1<p, q<\infty$ and $1 / p+1 / q=1$. If a compactly supported Borel probability measure $\mu$ has a $(p, q)$-Bessel/frame measure $\nu$, then it also has an atomic one. More precisely, if $\nu$ is a $(p, q)$-Bessel measure for $\mu$ and if $\nu^{\prime}$ is a discretization of the measure $\nu$, then $\nu^{\prime}$ is a $(p, q)$-Bessel measure for $\mu$.

If $\nu$ is a $(p, q)$-frame measure for $\mu$ and $r>0$ is small enough, then $\nu^{\prime}$ is a $(p, q)$-frame measure for $\mu$.

Proof. Let $Q=[0,1)^{d}$. Let $\left(x_{k}\right)_{k \in \mathbb{Z}^{d}}$ be a set of points such that $x_{k} \in r(k+Q)$ for all $k \in \mathbb{Z}^{d}$. For every $x \in r(k+Q)$ define $\epsilon(x):=x_{k}-x$. Thus, $|\epsilon(x)| \leq r \sqrt{d}=: r^{\prime}$ and for any $f \in L^{p}(\mu)$,

$$
\begin{aligned}
\int_{\mathbb{R}^{d}}\left|\left[f, e_{x+\epsilon(x)}\right]_{L^{p}(\mu)}\right|^{q} d \nu(x) & =\sum_{k \in \mathbb{Z}^{d}} \int_{r(k+Q)}\left|\left[f, e_{x_{k}}\right]_{L^{p}(\mu)}\right|^{q} d \nu(x) \\
& =\sum_{k \in \mathbb{Z}^{d}} \nu(r(k+Q))\left|\left[f, e_{x_{k}}\right]_{L^{p}(\mu)}\right|^{q} .
\end{aligned}
$$

Since we have

$$
\begin{aligned}
\int_{\mathbb{R}^{d}} \inf _{|y| \leq r^{\prime}}\left|\left[f, e_{x+y}\right]_{L^{p}(\mu)}\right|^{q} d \nu(x) & \leq \int_{\mathbb{R}^{d}}\left|\left[f, e_{x+\epsilon(x)}\right]_{L^{p}(\mu)}\right|^{q} d \nu(x) \\
& \leq \int_{\mathbb{R}^{d}} \sup _{|y| \leq r}\left|\left[f, e_{x+y}\right]_{L^{p}(\mu)}\right|^{q} d \nu(x),
\end{aligned}
$$

the upper and lower bounds follow from Theorem 4.3.

By Lemma 3.1, if there exists a purely atomic $(p, q)$-frame measure $\nu$ for a probability measure $\mu$, then there exists a $q$-frame for $L^{p}(\mu)$. Now we conclude that if there exists a $(p, q)$-frame measure $\nu$ (not necessarily purely atomic) for a compactly supported probability measure $\mu$, then there exists a $q$-frame for $L^{p}(\mu)$.

Corollary 4.2. Let $\mu$ be a compactly supported Borel probability measure. Let $1<p, q<\infty$ and $1 / p+1 / q=1$. If $\nu$ is a $(p, q)$-frame measure for $\mu$ with bounds $A, B$ and $r>0$ is sufficiently small, then there exist positive constants $C, D$ such that $\left\{c_{k} e_{x_{k}}: k \in \mathbb{Z}^{d}\right\}$ is a $q$-frame for $L^{p}(\mu)$ with bounds $C, D$, where $x_{k} \in r(k+Q)$ and $c_{k}=\sqrt[q]{\nu(r(k+Q))}$. 
Proof. Let $\nu \in \mathcal{F}_{A, B}(\mu)_{p, q}$. Then by Theorems 4.4 and $4.3, \nu^{\prime}=\sum_{k \in \mathbb{Z}^{d}} c_{k}^{q} \delta_{x_{k}}$ is a $(p, q)$-frame measure for $\mu$. More precisely, $\nu^{\prime} \in \mathcal{F}_{C, D}(\mu)_{p, q}$. Hence for all $f \in L^{p}(\mu)$,

$$
\begin{aligned}
C\|f\|_{L^{p}(\mu)}^{q} & \leq \int_{\mathbb{R}^{d}}\left|\left[f, e_{t}\right]_{L^{p}(\mu)}\right|^{q} d \nu^{\prime}(t)=\sum_{k \in \mathbb{Z}^{d}} c_{k}^{q}\left|\left[f, e_{x_{k}}\right]_{L^{p}(\mu)}\right|^{q} \\
& =\sum_{k \in \mathbb{Z}^{d}}\left|\left[f, c_{k} e_{x_{k}}\right]_{L^{p}(\mu)}\right|^{q} \leq D\|f\|_{L^{p}(\mu)}^{q} .
\end{aligned}
$$

\section{Acknowledgements}

The authors would like to thank Dr. Nasser Golestani for his valuable guidance and helpful comments.

\section{RE F E R E N C E S}

1. P. G. Casazza, O. Christensen and D. T. Stoeva: Frame expansions in separable Banach spaces J. Math. Anal. Appl. 307 (2005) 710-723.

2. O. Christensen: An Introduction to Frames and Riesz Bases. Applied and Numerical Harmonic Analysis, Birkhäuser Boston Inc., Boston, MA, 2003.

3. D. Dutkay, D. Han, Q. Sun and E. Weber: On the Beurling dimension of exponential frames. Adv. Math. 226 (2011) 285-297.

4. D. Dutkay, D. Han and E. Weber: Bessel sequence of exponential on fractal measures. J. Funct. Anal. 261 (2011) 2529-2539.

5. D. Dutkay, D. HAN and E. WeBer: Continuous and discrete Fourier frames for fractal measures. Trans. Amer. Math. Soc. 366 (3) (2014) 1213-1235.

6. D. DutKAy and P. JoRgEnSEn: Fourier frequencies in affine iterated function systems. J. Funct. Anal. 247 (1) (2007) 110-137.

7. D. DutKAY and C.-K. LAI: Self-affine spectral measures and frame spectral measures on $\mathbb{R}^{d}$. Preprint (2015). arXiv:1502.03209.

8. D. Dutkay and C.-K. Lai: Uniformity of measures with Fourier frames. Adv. Math. 252 (2014) 684-707.

9. D. Dutkay, C.-K. LAI and Y. Wang: Fourier bases and Fourier frames on self-affine measures. Preprint (2016). arXiv:1602.04750.

10. G. B. Folland: Real analysis. second ed., John Wiley, New York, 1999.

11. B. Fuglede: Commuting self-adjoint partial differential operators and a group theoretic problem. J. Funct. Anal. 16 (1974) 101-121.

12. J. R. Giles: Classes of semi-inner product spaces. Trans. Amer. Math. Soc. 129 (1967) 436-446.

13. X.-G. He, C.-K. LAI and K.-S. LAU: Exponential spectra in $L^{2}(\mu)$. Appl. Comput. Harmon. Anal. 34 (3) (2013) 327-338. 
14. T.-Y. Hu, K.-S. LAU and X.-Y. WANG: On the absolute continuity of a class of invariant measures. proc. Amer. Math. Soc. 130 (3) (2001) 759-767.

15. J. E. Hutchinson: Fractals and self-similarity. Indiana Univ. Math. J. 30 (5) (1981) 713-747.

16. P. Jorgensen and S. Pedersen: Dense analytic subspaces in fractal $L^{2}$-spaces. J. Anal. Math. 75 (1998) 185-228.

17. Y. Katznelson: An introduction to harmonic analysis. third ed., Cambridge Mathematical Library, Cambridge University Press, Cambridge, 2004.

18. I. LABA and Y. WAng: On spectral Cantor measures. J. Funct. Anal. 193 (2002) 409-420.

19. I. LABA and Y. WAng: Some properties of spectral measures. Appl. Comput. Harmon. Anal. 20 (1) (2006) 149-157.

20. C.-K. LAI: On Fourier frame of absolutely continuous measures. J. Funct. Anal. 261 (10) (2011) 2877-2889.

21. N. LEV: Fourier frames for singular measures and pure type phenomena. proc. Amer. Math. Soc. 146 (2018) 2883-2896.

22. G. Lumer: Semi-inner product spaces. Trans. Amer. Math. Soc. 100 (1961) 2943.

23. S. Nitzan, A. OlevskiI and A. UlanovskiI: Exponential frames on unbounded sets. Proc. Amer. Math. Soc. 144 (1) (2016) 109-118.

24. J. Ortega-Cerdì and K. Seip: Fourier frames. Ann. of Math. 155 (3) (2002) 789-806.

25. N. K. SAhu and R. N. Mohapatra: Frames in semi-inner product spaces. In: P. N. Agrawal, R. N. Mohapatra, Uaday Singh, H. M. Srivastava (Eds.), Springer Proceedings in Mathematics and Statistics 143, Mathematical analysis and its applications, Springer, New Delhi, 2015, pp. 149-158.

26. H. Zhang and J. ZhANG: Frames, Riesz bases, and sampling expansions in Banach spaces via semi-inner products. Appl. Comput. Harmon. Anal. 31 (2011) 1-25.

Fariba Zeinal Zadeh Farhadi Department of Mathematics Science and Research Branch Islamic Azad University, Tehran, Iran

fz.farhadi61@yahoo.com

Mohammad Sadegh Asgari

Department of Mathematics

Faculty of Science

Islamic Azad University

Central Tehran Branch, Tehran, Iran

moh.asgari@iauctb.ac.ir 
Mohammad Reza Mardanbeigi

Department of Mathematics

Science and Research Branch

Islamic Azad University, Tehran, Iran

mrmardanbeigi@srbiau.ac.ir

Mahdi Azhini

Department of Mathematics

Science and Research Branch

Islamic Azad University, Tehran, Iran

m.azhini@srbiau.ac.ir 